\title{
Nacre-inspired composites with different macroscopic dimensions: strategies for improved mechanical performance and applications
}

\author{
Hewei Zhao', Zhao Yang ${ }^{1}$ and Lin Guo ${ }^{1}$
}

\begin{abstract}
To develop next-generation lightweight, high-strength, and tough materials, new materials design strategies must be established. Nacre, consisting of 95 vol.\% inorganic plates $\left(\mathrm{CaCO}_{3}\right)$ and 5 vol.\% organic matrix (protein) in layered arrangements, is famous for its significant increase (three orders of magnitude higher) in toughness compared to monolithic aragonite and has always been the model for the synthesis of high mechanical performance artificial materials. In this review, we primarily introduce the recent studies on the synthesis of nacre-inspired composites with exceptional mechanical properties, including 1D fibers, 2D films, and 3D bulk materials. In addition, design strategies for performance enhancement are summarized based on these studies, and applications of high-performance nacreinspired composites are also discussed. Finally, a critical outlook of the future direction of developing next-generation high mechanical performance nacre-inspired composites is provided.
\end{abstract}

\section{INTRODUCTION}

One of the most ambitious goals in structural materials is to achieve exceptional mechanical properties, especially for an ideal combination of light weight, high strength, and high toughness ${ }^{1}$. Micro/nanostructured materials have constantly been studied since the 1990s, and some of these show remarkable mechanical properties, such as carbon nanotubes ${ }^{2}$, graphene ${ }^{3}$, nanometallic glass ${ }^{4}$, and ceramic nanoparticles ${ }^{5}$. However, only materials on the macroscopic scale can be applied to replace conventional structural materials. Hence, how to assemble micro/ nanomaterials on the macroscopic scale without sacrificing their mechanical properties is vital for fabricating next-generation lightweight and high mechanical performance materials. For many biomaterials, strategies to achieve the advantageous unification between structure and bulk properties have been through millions of years of

\footnotetext{
Correspondence: Lin Guo (guolin@buaa.edu.cn)

${ }^{1}$ School of Chemistry, Beijing Advanced Innovation Center for Biomedical Engineering, Beihang University, Beijing 100191, China
}

evolution ${ }^{6}$. Nacre, the inner layer of shells, is one of the most outstanding examples of combining high strength and high toughness in a lightweight fashion, as the brickand-mortar structure is considered the primary contributor to its marvelous mechanical properties ${ }^{7,8}$. Thus, mimicking nacre's micro/nanostructure in macroscopic artificial materials is an alternative way for scientists and engineers to fabricate high mechanical performance structural materials.

During the past few decades, many macroscopic structural materials with nacre-inspired uniform micro/ nanoarchitecture have been successfully synthesized, and their mechanical properties and functional applications have also been studied ${ }^{9}$. Since 2009, some reviews have been published to present the progress of nacre-inspired composites. Luz and Mano ${ }^{10}$ summarized the synthetic design strategies of nacre-inspired composites in 2009 and recently discussed their biomedical applications ${ }^{11}$. Mayer $^{12}$ reviewed the mechanisms underlying the toughening in rigid natural composites. Cheng et al. ${ }^{8}$

\section{(c) The Author(s) 2018}

(c) (i) Open Access This article is licensed under a Creative Commons Attribution 4.0 International License, which permits use, sharing, adaptation, distribution and reproduction cc) in any medium or format, as long as you give appropriate credit to the original author(s) and the source, provide a link to the Creative Commons license, and indicate if changes were made. The images or other third party material in this article are included in the article's Creative Commons license, unless indicated otherwise in a credit line to the material. If material is not included in the article's Creative Commons license and your intended use is not permitted by statutory regulation or exceeds the permitted use, you will need to obtain permission directly from the copyright holder. To view a copy of this license, visit http://creativecommons.org/licenses/by/4.0/. 


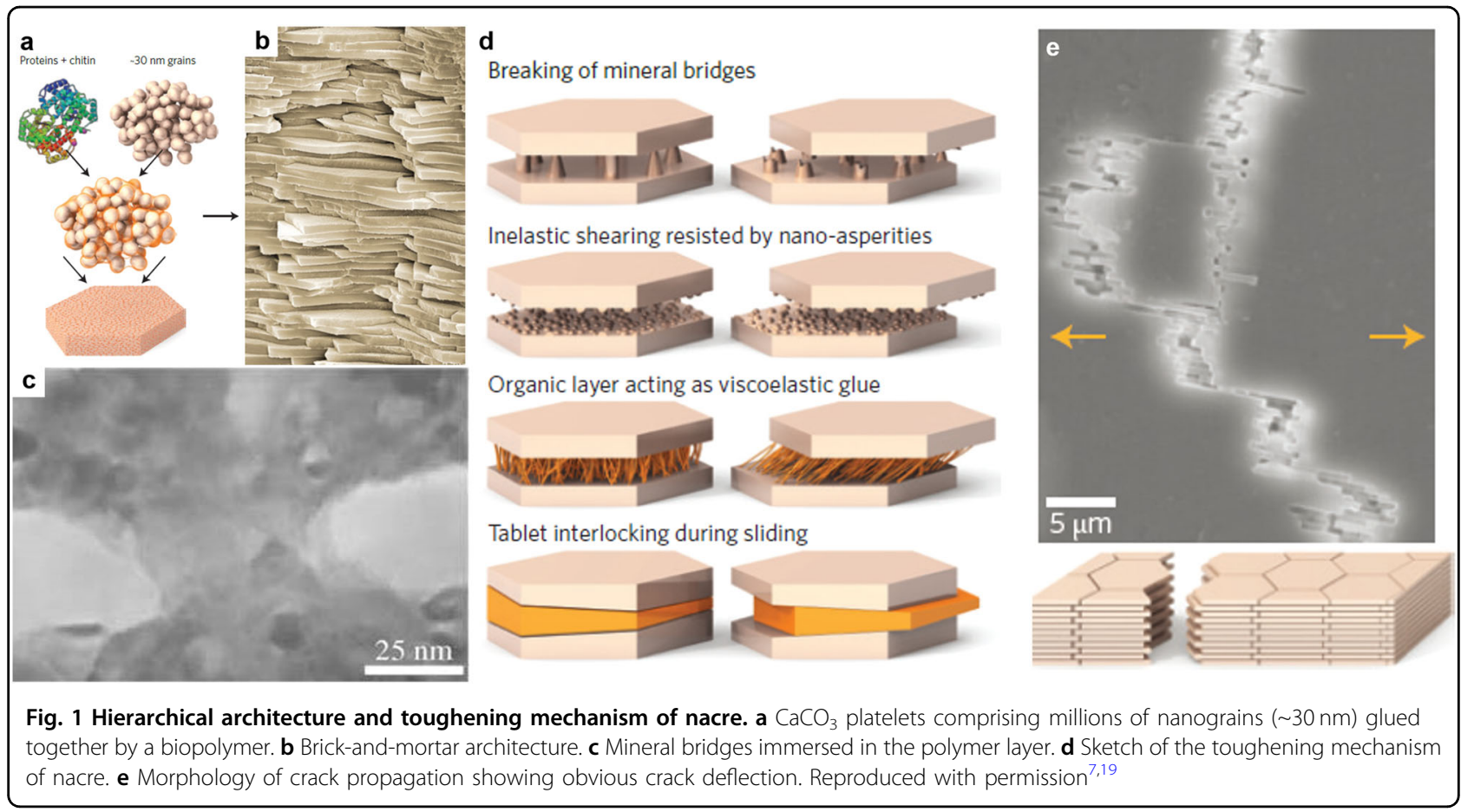

reviewed the preparation of layered organic-inorganic nanocomposites inspired by nacre and discussed the advantages and disadvantages of various biomimetic strategies. Meyers et al. ${ }^{13}$ reviewed all bio-inspired materials that show outstanding mechanical properties and focused on the relationships between structure and properties. Yu et al. ${ }^{9}$ summarized the synthesis, fabrication, and applications of nacre-inspired layered nanocomposites and focused on the biomineralization process. Ritchie et al. $^{7}$ reviewed high-performance structural materials with bio-inspired architecture and discussed the advantages of bio-mimetics. Cheng et al. ${ }^{14}$ focused on graphene-based nacre-inspired composites, which can effectively take advantage of graphene's excellent mechanical properties. Grunlan et al. ${ }^{6}$ summarized the synthetic methods for mimicking different kinds of natural structures at the micro/nanoscale, and the recent progress in nacre-inspired composites was also mentioned. As an important factor in influencing the mechanics of biological materials, interfaces of nacre, bone and wood were discussed by Barthelat et al. and inspired design strategies for recently highlighted synthetic biomaterials ${ }^{15}$. Moreover, the comparison of biologically inspired materials with those prepared using additive manufacturing was reviewed by Studart et al. ${ }^{16}$.

The reviews mentioned above are mainly focused on the methods to construct brick-and-mortar structure in artificial materials, the mechanisms underlying relationships between structure and properties and the applications in some fields. However, the mechanical properties of conventional nacre-inspired composites are reaching their limit, as only mimicking nacre's brick-and-mortar structure makes a limited contribution to further enhancing the performance of such materials ${ }^{14}$. Strategies for further improving the mechanical properties of nacre-inspired composites are not obvious but are in great demand. To summarize those strategies, the nacre-inspired composites in this review were categorized by dimensions, as the conditions to realize their functions must be considered when using these structural materials; for example, cables need one dimensional (1D) fibers, coatings need twodimensional (2D) films, and main bearing members need three dimensional (3D) bulk materials. Nevertheless, reviews that summarize the nacre-inspired composites with different dimensions and that discuss the advantages of different dimensional materials are uncommon.

In this review, we overview the recent achievements in the synthesis of high-performance nacre-inspired composites and divide them into different groups based on their dimensions, such as 1D nacre-inspired fibers, 2D nacre-inspired films, and 3D nacre-inspired bulk composites. The methods to produce different dimensional nacre-inspired composites are also introduced, and performance-enhancing strategies for different dimensional nacre-inspired composites are summarized and explained in detail. Applications of high mechanical performance nacre-inspired composites are also summarized. Finally, we provide a critical outlook of the development of next-generation high mechanical property materials by increasing interfacial interactions and optimizing the 


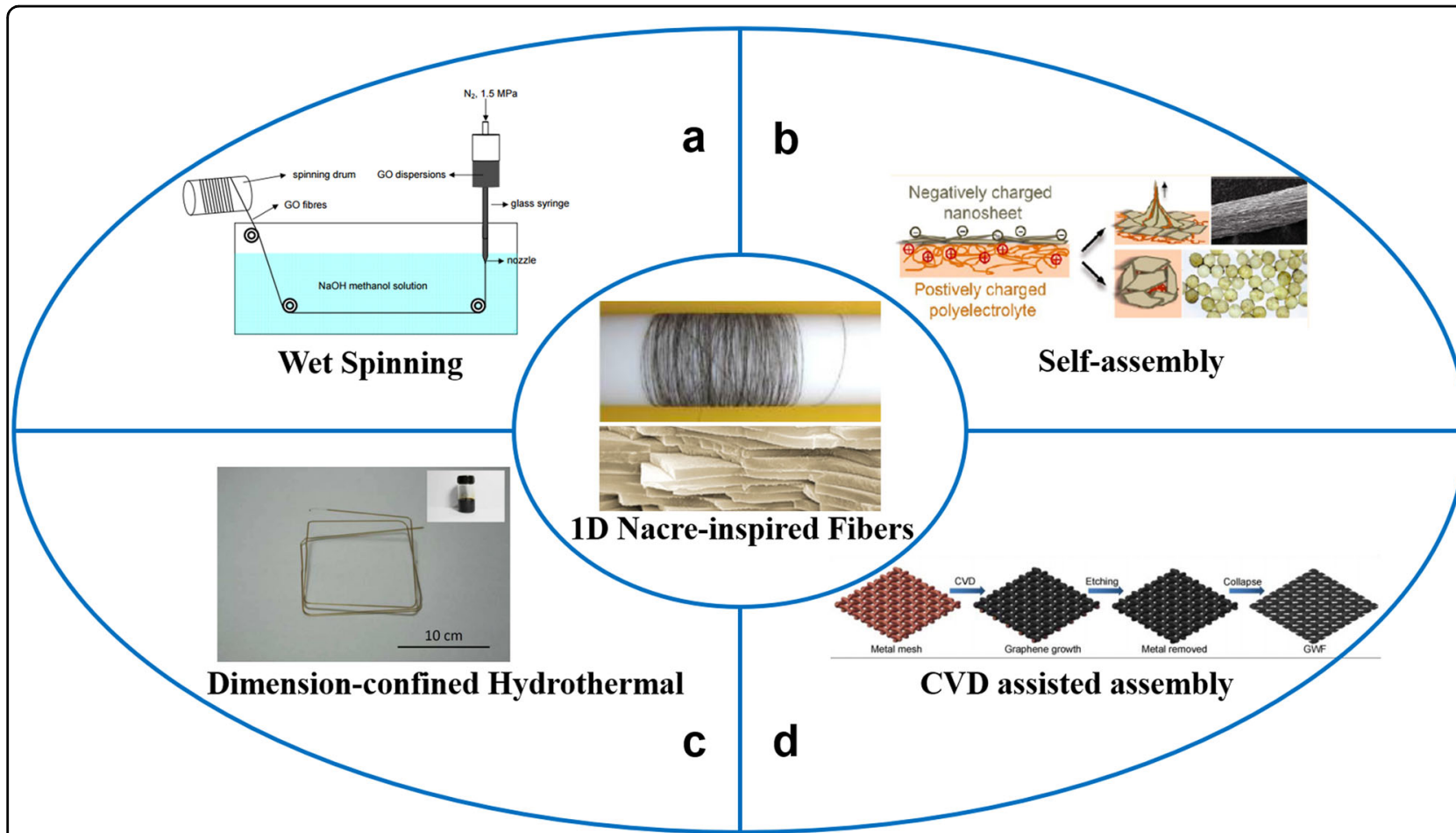

Fig. 2 Typical methods for synthesizing 1D nacre-inspired fibers. a Wet spinning. Reproduced with permission ${ }^{31}$. b Self-assembly. Reproduced with permission ${ }^{32}$. c Dimensionally confined hydrothermal treatment. Reproduced with permission ${ }^{34}$. $\mathbf{d}$ Chemical vapor deposition (CVD)-assisted assembly. Reproduced with permission ${ }^{33}$

micro/nanoarchitecture, which will better satisfy the demand for high-speed development of engineering and industrial researchers.

\section{Learning from nacre}

Nacre shows a hierarchical layered architecture: a stacked structure composed of $95 \mathrm{vol} . \%$ aragonite calcium carbonate platelets and 5 vol.\% organic (proteins and chitin) lamellae (Fig. 1) $)^{7,17}$. In nacre, the calcium carbonate platelets (width of $5-8 \mu \mathrm{m}$, thickness of $0.5 \mu \mathrm{m}$ ) serve as the brick (Fig. 1b) by providing strength, and the organic layer (thickness: $20-30 \mathrm{~nm}$ ) serves as the mortar (Fig. 1b) by providing ductility capacity and energy redistribution at the microscale; together, they generate nacre's excellent mechanical properties ${ }^{17}$. Close investigation of the structure of nacre showed that the calcium carbonate platelets comprise millions of nanograins ( 30 $\mathrm{nm}$ ) glued together by a biopolymer (Fig. 1a) at the nanoscale ${ }^{18}$ and that there are numerous mineral bridges encompassed in the organic layer (Fig. 1c) at the mesoscale $^{19}$. It is obvious that the hierarchical architecture has two levels: calcium carbonate nanograins are glued together to form the calcium carbonate platelets, and the calcium carbonate platelets are stacked together to form the brick-and-mortar structure.
The mechanical properties of nacre have been carefully characterized by some modern testing methods, such as tensile tests $^{20}$, compressive tests ${ }^{21}$, bending tests ${ }^{17}$, shearing tests ${ }^{17}$, and nanoindentation ${ }^{22}$. The tensile strength and modulus of nacre along the lamellar direction are $80-130 \mathrm{MPa}$ and $60-70 \mathrm{GPa}$, respectively ${ }^{23}$. In particular, the toughness of nacre is up to $1.24 \mathrm{~kJ} / \mathrm{m}^{2}$, which is three orders of magnitude higher than that of calcium carbonate ${ }^{24}$.

The hierarchical layered architecture of nacre mainly contributes to its impressive improvement in toughness without sacrificing strength ${ }^{17}$. When force is applied to nacre, the hierarchical structure generates limited deformation at the nanoscale to redistribute the energy as presented in Fig. 1d, including the breaking of mineral bridges between calcium carbonate platelets, shearing resistance contributed by nano-asperities, viscoelastic glue provided by the organic layer and tablet interlocking ${ }^{25}$. The mechanical behaviors mentioned above will generate crack deflection at the microscale during crack propagation (Fig. 1e), which dissipates much more energy than a straight crack propagation path ${ }^{26}$. Crack tip deformation $^{26}$, crack blunting $^{27}$, and platelet pull-out ${ }^{13}$ also occur during crack propagation to toughen nacre. In addition, recent research ${ }^{28}$ demonstrated that the brittle calcium 


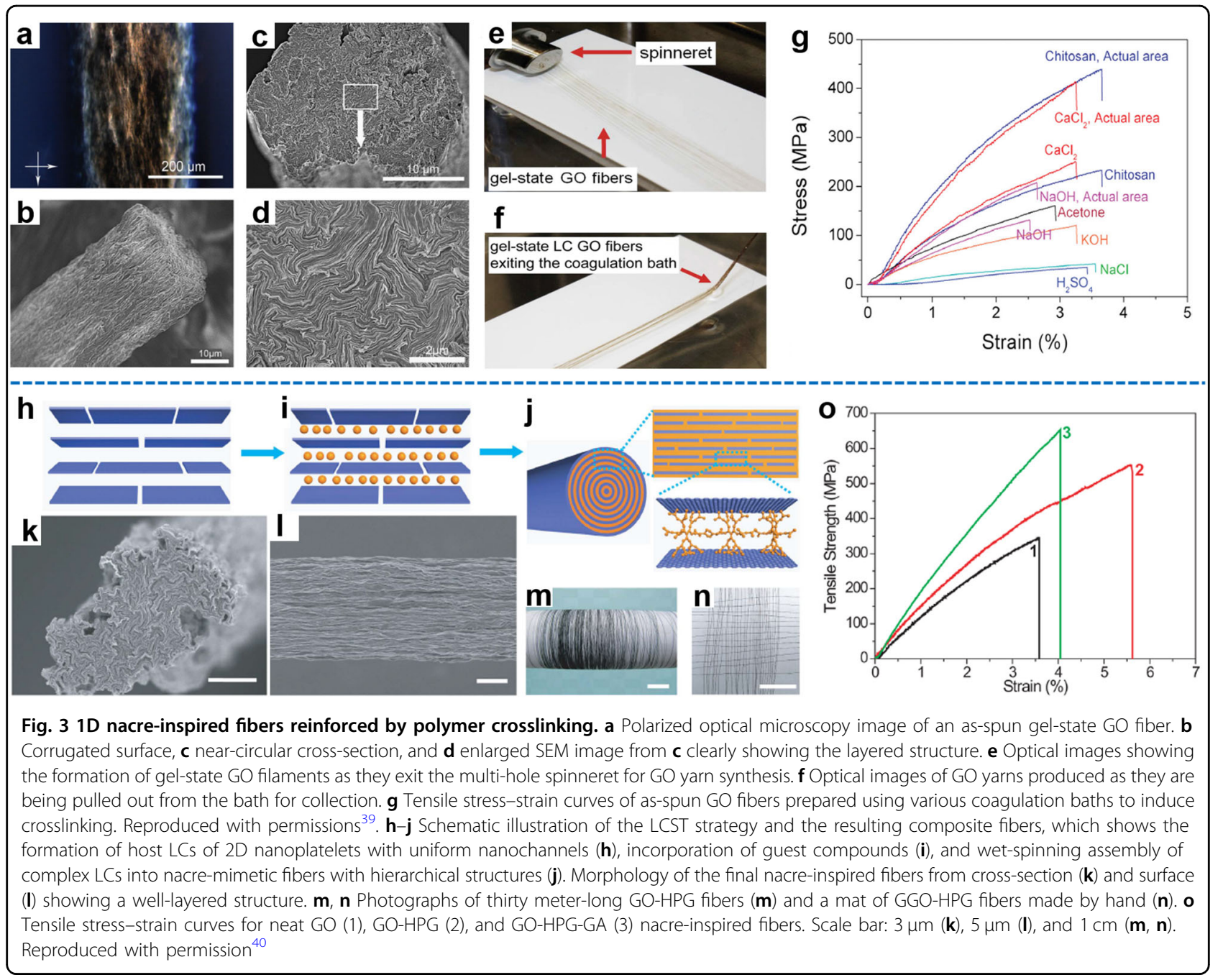

carbonate platelets can bend to some degree to further improve nacre's toughness.

\section{Nacre-inspired composites \\ 1D nacre-inspired fibers}

1D fibers are widely used in modern society, such as for making yarns for clothes, for twisting into ropes to transfer a mechanical load, and for conducting electricity to transfer electric power ${ }^{29}$. However, the currently used materials cannot be strong and tough simultaneously; for instance, yarns are usually made from cotton fibers, which are tough but weak. 1D nacre-inspired fibers with high strength and high toughness have started to become an alternative to traditional fibers in various applications.

To date, graphene, which has extraordinary properties, is the material most widely used to synthesize $1 \mathrm{D}$ macroscopic fibers, and several methods have been developed, including wet spinning, self-assembly, dimensionally confined hydrothermal treatment, and chemical vapor deposition (CVD)-assisted assembly
(Fig. 2) ${ }^{30-34}$. For instance, $\mathrm{Xu}$ and $\mathrm{Gao}^{31}$ found that graphene oxide (GO)/reduced graphene oxide (rGO) nanosheets can form chiral liquid crystals (CLCs) in aqueous dispersions, which were subsequently precipitated into a methanol $/ \mathrm{NaOH}$ coagulation bath and transformed into continuous fibers using a continuous process (also called the wet-spinning method) for the first time in 2011 (Fig. 2a). The GO/rGO-based fibers show a microlayered structure and exhibit good mechanical performance (140 MPa at an ultimate elongation of 5.8\%). Later, wet spinning was demonstrated to be relatively easy to scale-up to multiple spinnerets by Jalili et al. ${ }^{35}$. Through the complexation of GO nanosheets, Zhou and $\mathrm{Kim}^{32}$ successfully introduced self-assembly behaviors similar to polyelectrolyte-polyelectrolyte systems to synthesize layered structures, which were subsequently drawn as a fiber by grabbing them with a tweezer and gently pulling them up (Fig. $2 \mathrm{~b}$ ). Qu et $\mathrm{al}^{33}$. developed an efficient one-step dimensionally confined hydrothermal method to fabricate graphene-based fibers with a layered 


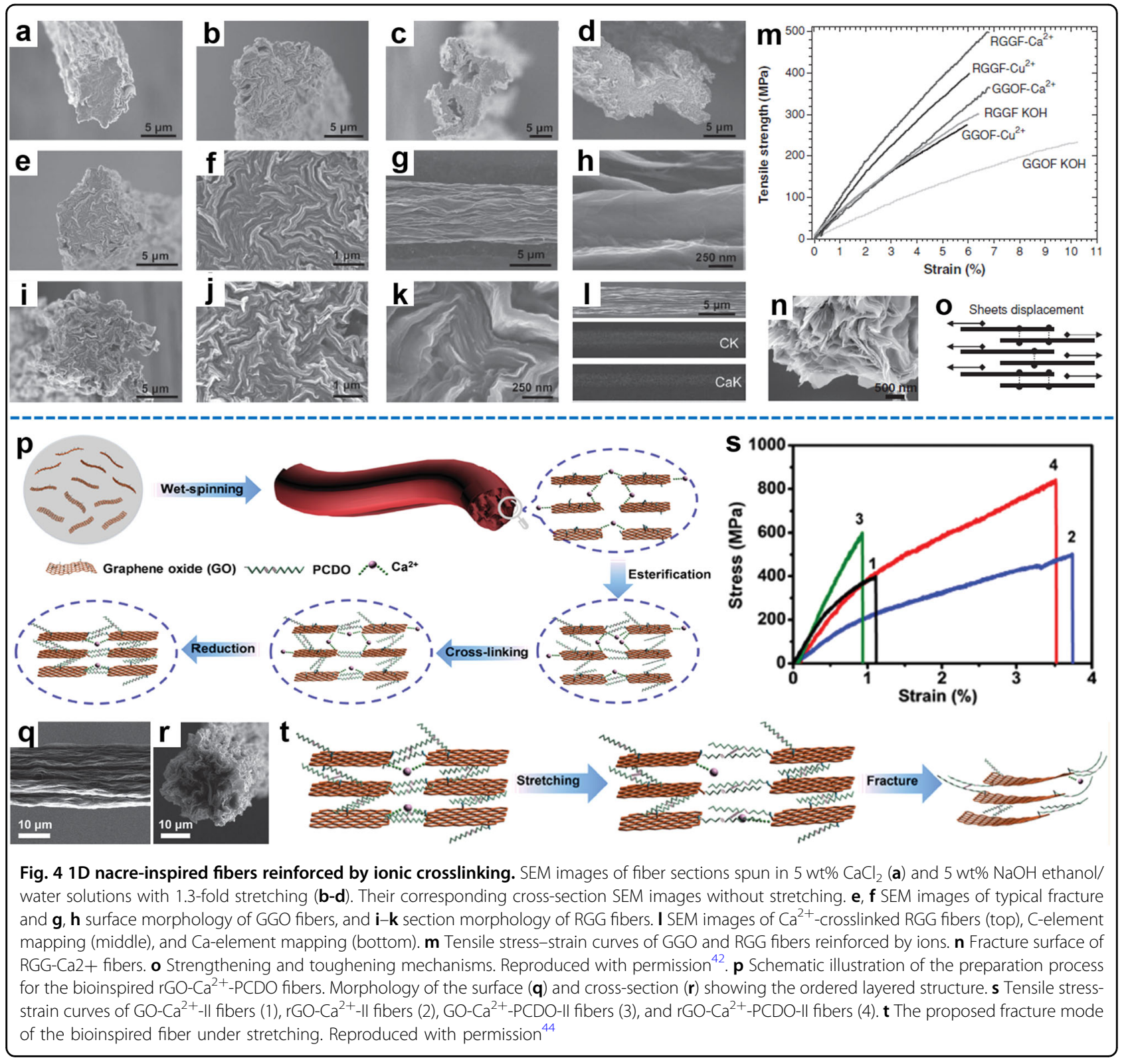

structure by reducing a concentrated GO dispersion hydrothermally within a cylindrical tube (Fig. 2c); the tensile strength of the resulting is up to $400 \mathrm{MPa}$ after thermal treatment at $800{ }^{\circ} \mathrm{C}$. Chemical vapor deposition (CVD)-assisted assembly is another alternative method to fabricate nacre-inspired 1D fibers (Fig. 2d). Zhu et $\mathrm{al}^{34}$. made graphene grow directly onto the surface of $\mathrm{Cu}$ wires by CVD, and graphene fibers can be synthesized after removing the $\mathrm{Cu}$.

Although the mechanical properties of 1D fibers can be improved by introducing a layered structure into the fibers through the methods mentioned above, the mechanical properties are limited owing to weak layer interactions, irregular layered structures and defects. The strategies reviewed below include polymer crosslinking and ionic crosslinking, which are efficient in increasing interfacial interactions, and twisting, which can remarkably increase the uniformity and decrease the defects of nacre-inspired fibers.

\section{Polymer crosslinking}

Polymer such as polyacrylonitrile ${ }^{36}$, poly(vinyl alcohol) $(\mathrm{PVA})^{37}$, and poly(glycidyl methacrylate) (PGMA) ${ }^{38}$ were introduced as the soft phase to layered graphene fibers to optimize their mechanical properties and strengthen the interfacial interactions. For instance, Jalili et al. ${ }^{39}$ developed a one-step wet-spinning method to synthesize graphene-based 1D fibers in coagulation baths with 


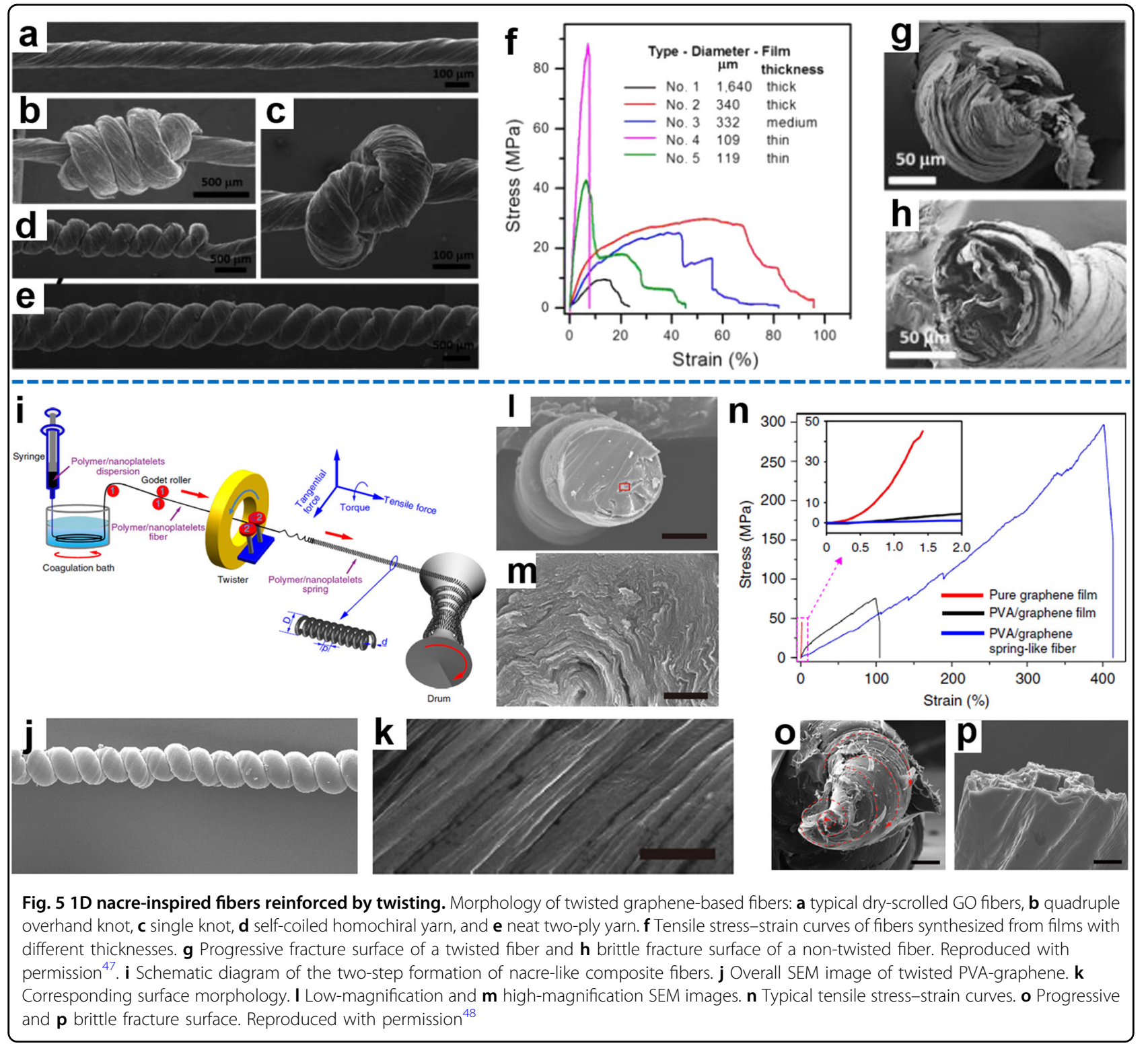

chitosan, as shown in Fig. 3e, f. The morphology of the nacre-inspired graphene chitosan fibers is shown in Fig. 3a-d. It is obvious that the fibers show a uniform layered structure (Fig. 3b, d). Chitosan is a positively charged polymer that can not only crosslink with GO but also participate in polyionic complexation. The enhanced interfacial interactions generated a great improvement in the mechanical performance of the graphene-based fibers, the Young's modulus, strength and toughness of which were up to $22 \mathrm{GPa}, 442 \mathrm{MPa}$, and $4.8 \mathrm{~J} / \mathrm{g}$, respectively (Fig. 3g), which are much higher than those of previously reported layered graphene fibers.

However, the polymer crosslinking process only occurred on the surface of the 1D fiber, a limitation of the coagulation bath, and the different polymer layers were independent of each other; thus, the enhancement of the mechanical properties was confined ${ }^{29}$. Gao et $\mathrm{al}^{40}$. combined a liquid crystal self-templating (LCST) approach (as shown in Fig. $3 \mathrm{~h}-\mathrm{j}$ ) with wet-spinning technology to continuously fabricate biomimetic composites. The LCST process could introduce a strong polymer binder (hyperbranched polyglycerol (HPG)) into the whole interface between GO layers (Fig. 3i), which could fully take advantage of the polymer's mechanical optimization. After subsequent wet spinning, 1D GO-HPG fibers were synthesized, which showed a uniform layered structure (Fig. 3k, l) similar to that in nacre. In addition, the length of the wet-spun fibers could be up to $30 \mathrm{~m}$ (Fig. $3 \mathrm{~m}, \mathrm{n}$ ), as the GO-based suspensions were more stable. The crosslinking between $\mathrm{GO}$ and the polymer and interactions 


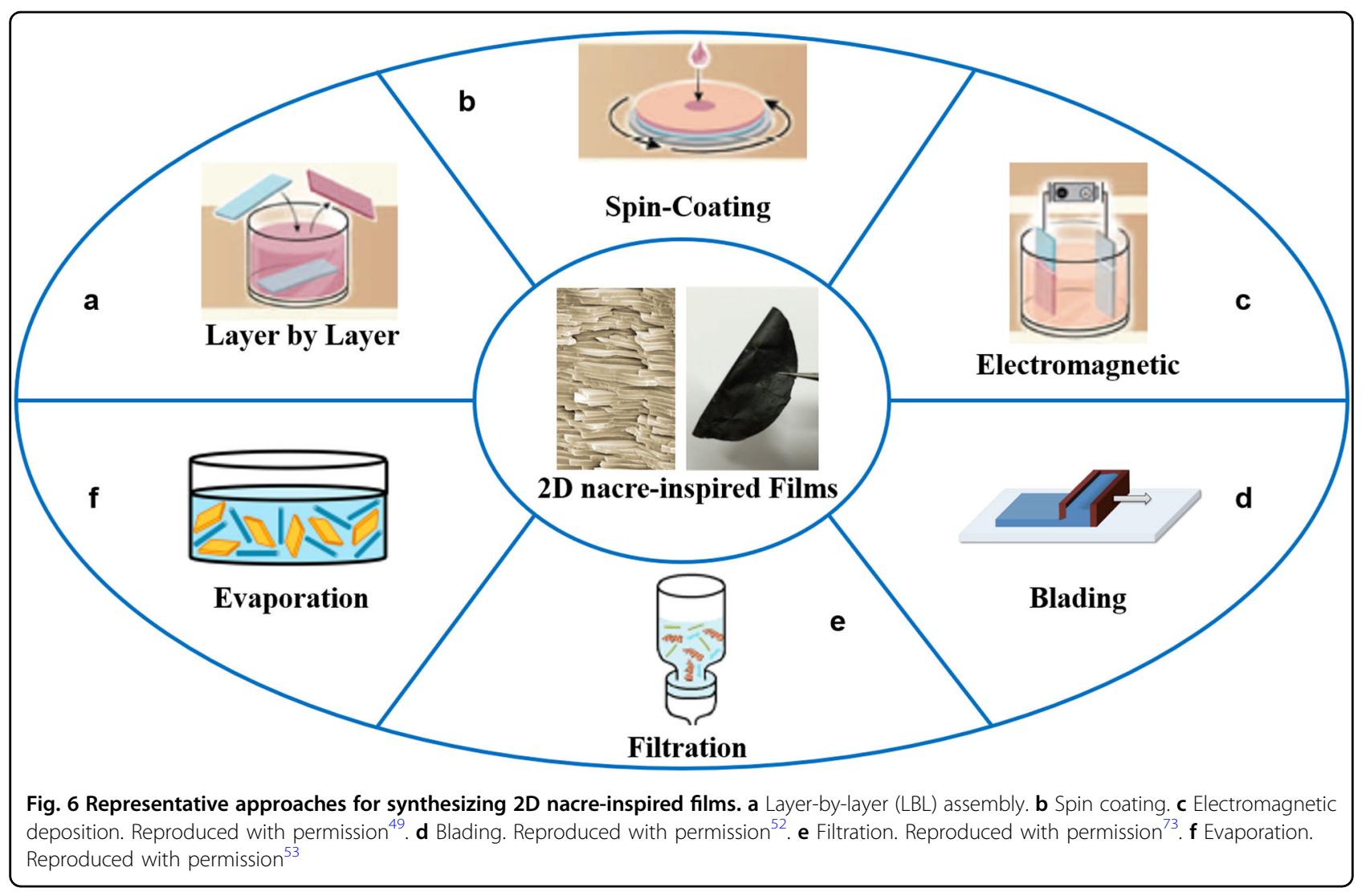

between layers were enhanced owing to the fully immersed polymer, generating an exceptional combination of strength and toughness, i.e., up to $555 \mathrm{MPa}$ and 18 $\mathrm{MJ} / \mathrm{m}^{3}$ (Fig. 3o), which were 4 times and over 10 times higher than those of nacre, respectively (strength of $80-130 \mathrm{MPa}$ and toughness of $0.1-1.8 \mathrm{MJ} / \mathrm{m}^{3}$ ). Furthermore, the strength of the fiber could be continuously promoted by introducing glutaraldehyde (GA) into the layered composites, which could crosslink with both HPG and GO to further improve the interlayer interactions. The fracture strength of the GO-HPG-GA fibers was 652 $\mathrm{MPa}$ (Fig. 3o), which was the highest at that time.

\section{Ionic crosslinking}

Based on the buried inorganic-organic interfaces derived from the various ions or ceramics in nacre, such as $\mathrm{Mg}^{2+}, \mathrm{Al}_{2} \mathrm{O}_{3}$, and $\mathrm{ZrO}_{2}{ }^{41}$, introducing metal ions into artificial nacre-inspired composites is another way to further improve their mechanical properties by enhancing interfacial interactions. Some divalent cations, e.g., $\mathrm{Ca}^{2+}$, $\mathrm{Mg}^{2+}$, and $\mathrm{Cu}^{2+}$, that have been demonstrated to readily react with carboxylate functional groups were adopted in the layered graphene fibers to increase the initial interfacial interaction ${ }^{42,43}$. For instance, Gao et al. ${ }^{42}$ successfully fabricated $\mathrm{Ca}^{2+} / \mathrm{Cu}^{2+}$-reinforced graphene-based fibers with a layered architecture by introducing the corresponding metal ions in the coagulation bath during the wet-spinning process. In addition, giant graphene oxide (GGO), which could efficiently reduce the defective edges of 1D fibers and make the layered structure more ordered, was used here to synthesize the layered fibers. As a result, the morphology of the synthesized fibers was indeed more uniform and ordered, as shown in Fig. 4a-l. EDS maps (Fig. 4l) demonstrated that $\mathrm{Ca}^{2+}$ was evenly distributed between the GGO layers. As expected, the $\mathrm{Ca}^{2}$ ${ }^{+}$-reinforced GGO fibers showed high strength (364 MPa) and high toughness (fracture strain of 6.8\%); the strength was 2 times higher than that of the fibers without $\mathrm{Ca}^{2+}$ reinforcement (Fig. 4m). Notably, after reduction of GO (RGG), the fracture strength of the $\mathrm{Ca}^{2+}$-reinforced RGG layered fibers was increased to over $500 \mathrm{MPa}$, a record value at that time.

Recently, Cheng et $\mathrm{al}^{44}$. successfully synthesized $\mathrm{Ca}^{2+}$-reinforced GO/rGO-pentacosadiyn-1-ol (PCDO) layered fibers by a combination of wet-spinning and dipcoating methods, as shown in Fig. 4p. $\mathrm{Ca}^{2+}$-reinforced GO fibers were synthesized first by introducing $\mathrm{Ca}^{2+}$ into the coagulation bath through wet spinning and subsequently combining it with PCDO through dip-coating. The final $\mathrm{rGO}-\mathrm{Ca}^{2+}$-PCDO hybrid fibers were synthesized after reducing GO (Fig. 4p). The morphology of the rGO- $\mathrm{Ca}^{2+}$-PCDO nacre-inspired fibers, which was 


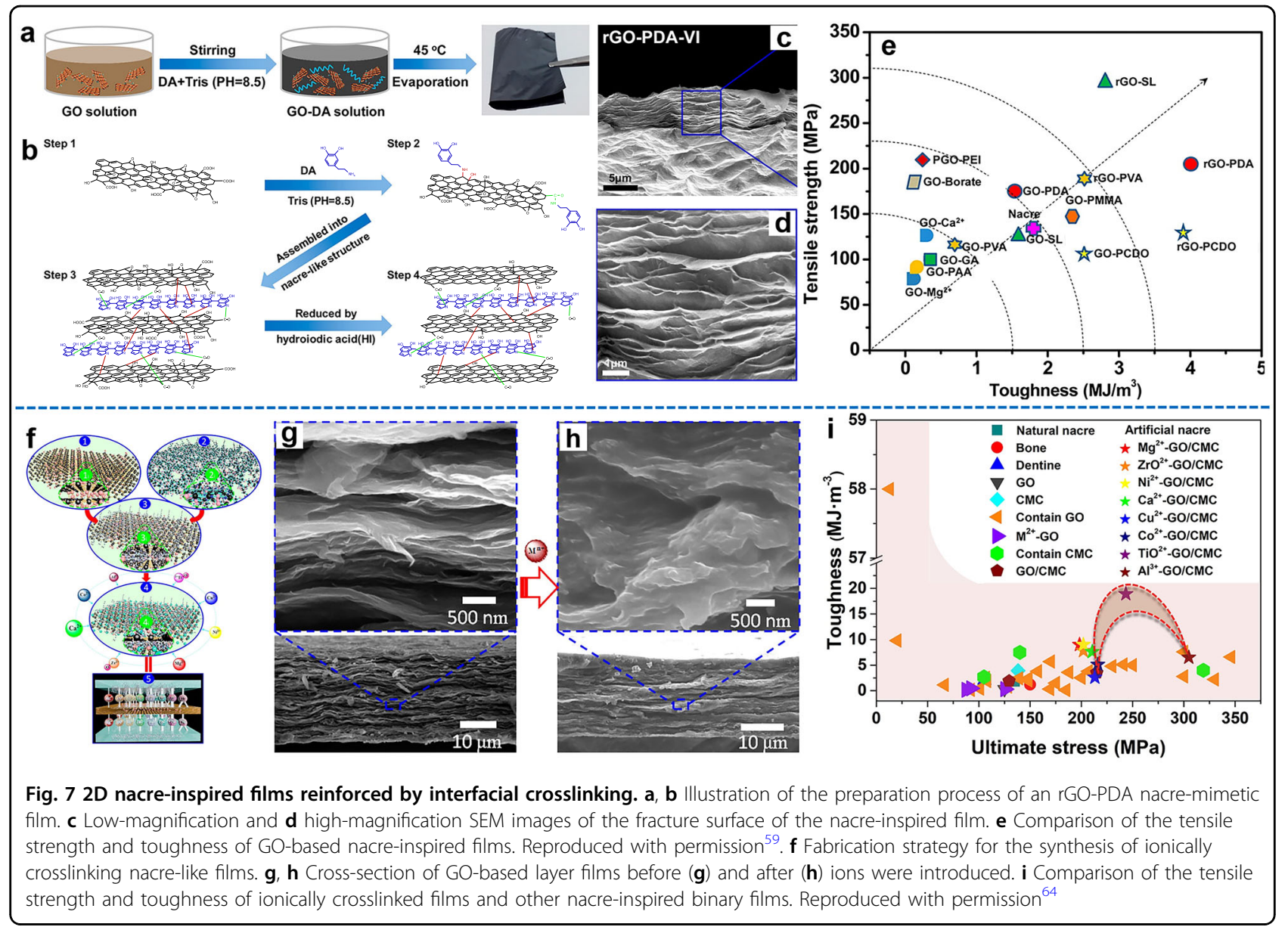

uniform and neat, is shown in Fig. 4q, r. The $\mathrm{Ca}^{2+}$-reinforced nacre-inspired fibers showed an ultrahigh strength of $842 \mathrm{MPa}$ and a high toughness of $16 \mathrm{MJ} / \mathrm{m}^{3}$ (Fig. $4 \mathrm{~s}$ ). In particular, the tensile strength of these novel fibers was the highest to date. Ionic bonding with $\mathrm{Ca}^{2+}$ and covalent bonding with $\mathrm{PCDO}$ in the hybrid fiber synergistically contributed to the outstanding improvement in its mechanical properties. When a load was applied to the fiber, the crosslinking caused by $\mathrm{Ca}^{2+}$ and PCDO restricted the sliding and pull-out of the $\mathrm{rGO}$ nanosheets (Fig. 4), which would dissipate a huge amount of energy and enhance the performance of the fibers.

\section{Twisting}

A clear disadvantage of 1D nacre-inspired fibers synthesized by the wet-spinning method is their rough surface, which generates self-abrasion and unwanted stress concentration under loading and results in unexpected breakage $^{45}$. Twisting is a common way to scroll carbon nanotubes into 1D fibers with fewer defects and a smooth surface $^{46}$. Introducing twisting to nacre-like layered graphene fibers may solve the problem of their rough surface. Based on twisting, Kim et $\mathrm{al}^{47}$. synthesized a film with enhanced toughness by bar coating water-based dispersions of GO followed by drying at room temperature and subsequently scrolled the film into fibers with a layered structure. The morphology and knot architecture of the fibers are shown in Fig. 5a-e, and it was obvious that the surface of the fibers synthesized by twisting was smooth. The mechanical properties depended on the thickness of the layered GO films before twisting (Fig. 5f), and the max elongation of the fiber is up to $80 \%$, superior to those of previously reported wet-spun fibers. The progressive fracture process of the twisting fiber (as shown in Fig. 5g), which resulted from the increased complexity of the hierarchical architecture compared to that of fibers synthesized by wet spinning (Fig. 5h), could dissipate much more energy to achieve excellent toughness and ductility.

Recently, a 1D graphene-PVA fiber with a nanoscale nacre-like brick-and-mortar structure and microscale twisting was successfully fabricated by Kotov et al. ${ }^{48}$. They developed a shear-induced self-assembly method combined with twisting technology (Fig. 5i) to synthesize the multiscale reinforced nacre-like fibers. The morphology of the fibers is shown in Fig. 5j-m. The uniform nacre-like layered structure (Fig. 5k) and smooth surface (Fig. 5l, m) 


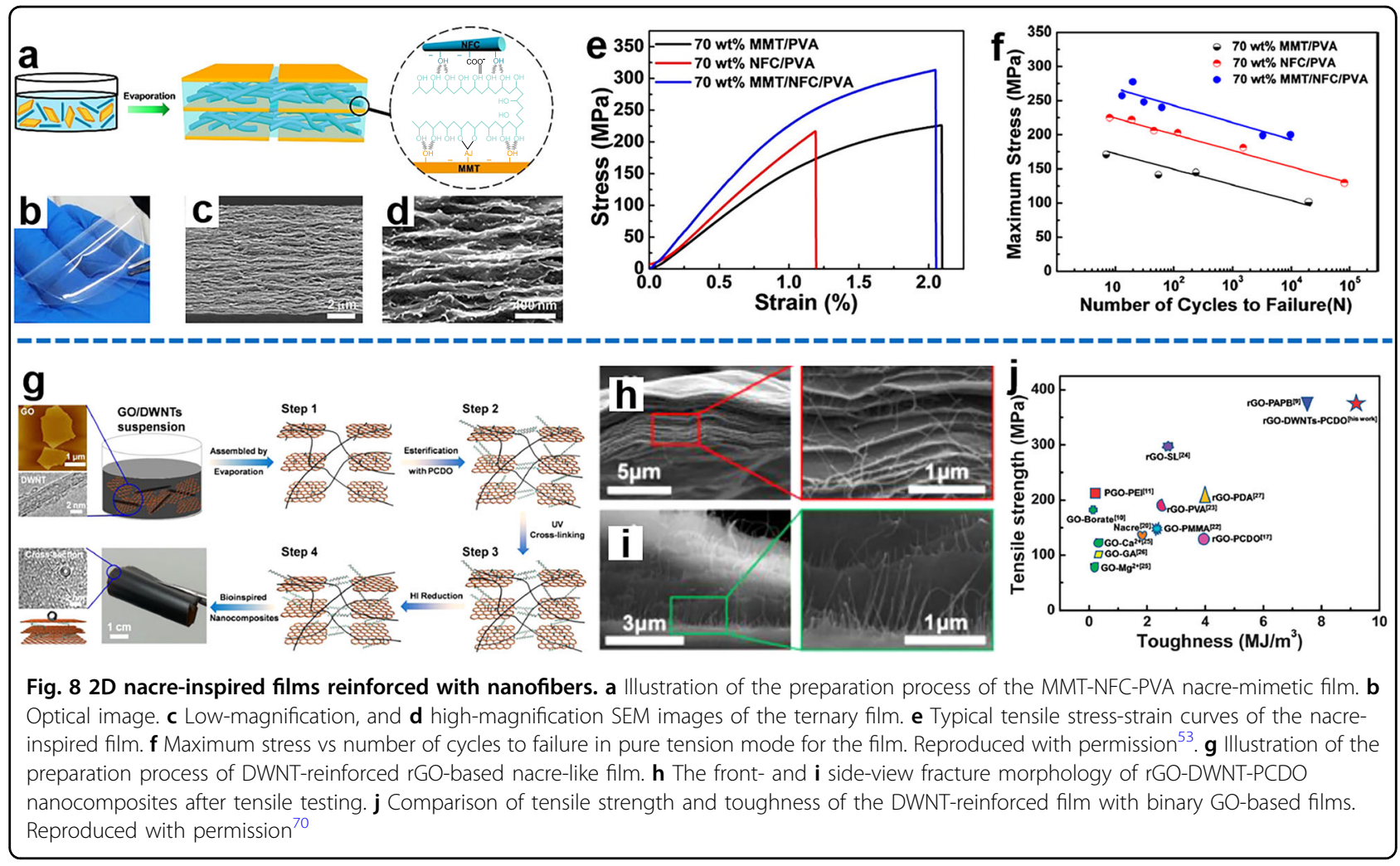

could be easily observed. After twisting, the fiber showed a spring-like macroscopic structure (Fig. 5j) and exhibited an exceptional elongation at break (over 400\%), 100 times and 4 times higher than those of pure graphene fibers and PVA-graphene fibers without the twisting structure, respectively. The fracture surface of the twisted nacre-like fiber indicated a progressive process (Fig. 5o), while the common nacre-like fiber with a belt-like structure showed a relatively flat fracture surface (Fig. 5p) The synergetic reinforcement between the nanoscale brick-and-mortar structure and macroscale twisted spring structure contributed to the marvelous toughness observed.

\section{D nacre-inspired films}

2D films have been linked to human civilization for several thousand years. Paper, which was invented during the Han dynasty in China to store information, is a typical 2D cellulose film. Today, the applications of films have been expanded to many fields, such as construction, industrial packaging, functional coatings, and flexible devices ${ }^{29}$. Unfortunately, the weak mechanical properties of traditional 2D films extremely restrict their further applications.

Nacre provides us with a way to synthesize high mechanical performance $2 \mathrm{D}$ films by introducing the brick-and-mortar layered structure into artificial materials. Through scientists' unremitting efforts, several assembly methods (Fig. 6) have been developed to produce nacre-inspired 2D films since $2003^{49-53}$. Kotov et al. ${ }^{54}$ successfully synthesized a clay/polymer-based nacre-like film by sequential adsorption of montmorillonite platelets (MTM) and polycationic poly(diallyldimethylammonium) chloride (PDDA) on a substrate. Cyclic repetition of the organic-adsorption/rinsing/inorganic-adsorption/rinsing process (usually called layer-bylayer assembly) resulted in a thin MTM-PDDA film with multiple layers (Fig. 6a). The nacre-like MTM-PDDA film showed a fracture strength and strain of $\sim 105 \mathrm{MPa}$ and $1 \%$, respectively, which are approximately equal to those of nacre. The mechanical properties of nacre-like claybased films synthesized by LBL assembly could be promoted by modifying the $\mathrm{MTM}^{55}$ and crosslinking the polymer $^{56}$. To further arrange the layer structure of hybrid films synthesized by LBL assembly, centrifugal force ${ }^{50}$ or electric/magnetic attraction ${ }^{51}$ was introduced in the LBL process as new methods called dip-coating (Fig. 6b) and electromagnetic deposition (Fig. 6c), respectively. However, an obvious disadvantage of the LBL method is that it is time-consuming; thus, films synthesized by LBL assembly are limited to sub- $5 \mu \mathrm{m}$ thicknesses ${ }^{8}$. To effectively increase the thickness of nacre-inspired films, some self-assembly methods such as blading $^{52,57}$ (Fig. 6d), filtration ${ }^{51}$ (Fig. 6e), and evaporation $^{53,58}$ (Fig. 6f) were developed in the following years. For instance, clay nanosheets (MTM) coated with a soft polymer (PVA) were driven to rapidly self-assemble into 


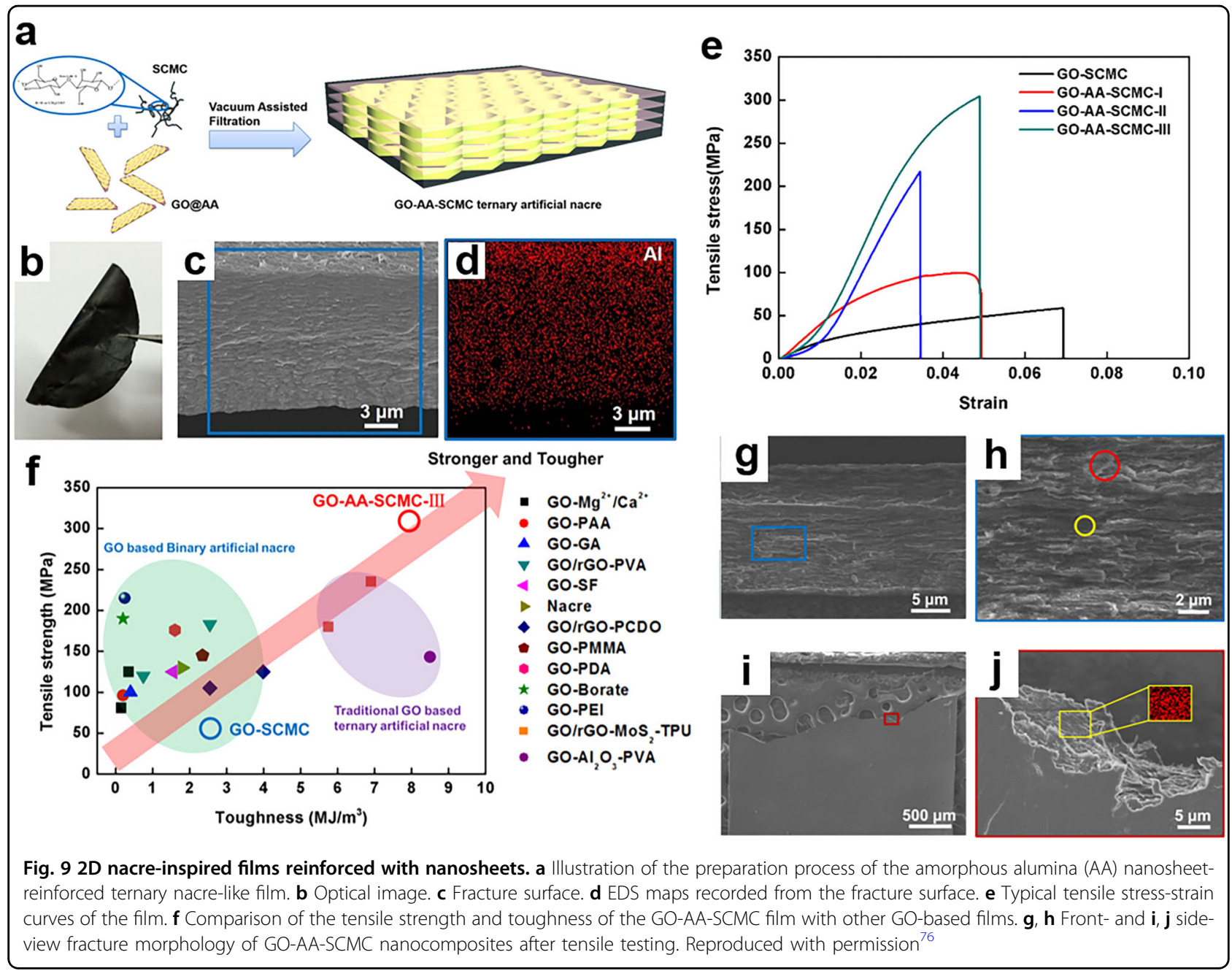

aligned nacre-like films by a filtration method $^{51}$. The thickness of the film could reach over $200 \mu \mathrm{m}$, which was dozens of micrometers thicker than films fabricated by LBL assembly. The fracture strength and strain of the MTM-PVA film were approximately $160 \mathrm{MPa}$ and $1 \%$, respectively, which are superior to those of nacre.

The development of 2D nacre-inspired films has consistently focused on mimicking nacre's brick-and-mortar structure in artificial materials, yet the complex interfacial interaction in nacre contributing to its remarkable mechanical properties should not be ignored ${ }^{9}$. Some synergistic interactions generated by biomolecules, ions, 1D nanofibers or 2D nanosheets have been demonstrated to be able to promote the mechanical properties of layered 2D nacre-like films, which are reviewed in detail in the following section.

\section{Interfacial crosslinking}

Dopamine (DA) is a typical biomolecule involved in mussel adhesives that contains both catechol and amine functional groups. Cui et al. ${ }^{59}$ adopted dopamine as a soft phase and combined it with GO to synthesize a layered nacre-like film through an evaporation method (Fig. 7a). The advantages of using DA was that DA can not only react with GO to form covalent bonds but also selfpolymerize into long-chain polymers of poly(dopamine) (PDA) through its abundant functional groups (Fig. 7b). The strong interfacial crosslinking resulted in the curving of the GO sheets in the hybrid film (Fig. 7c, d). The synergistic effects of covalent bonding and hydrogen crosslinking between GO and PDA simultaneously increased interfacial interactions and contributed to the film's exceptional mechanical properties. After reduction by $\mathrm{HI}$, the rGO-PDA nacre-like film showed a tensile strength of $204 \mathrm{MPa}$ and a toughness of $4 \mathrm{MJ} / \mathrm{m}^{3}, 1.5$ and 2 times higher than those of nacre, respectively (Fig. 7e). Some other organic molecules, such as silk fibroin $(\mathrm{SL})^{60}$, chitosan $^{61}$, and a combination of 1-aminopyrene and disuccinimidyl suberate (AP-DSS) ${ }^{62}$, were also introduced into nacre-inspired films to enhance their mechanical properties. For instance, the rGO-SL layered films showed a strength as high as $300 \mathrm{MPa}$, which was a record value at 


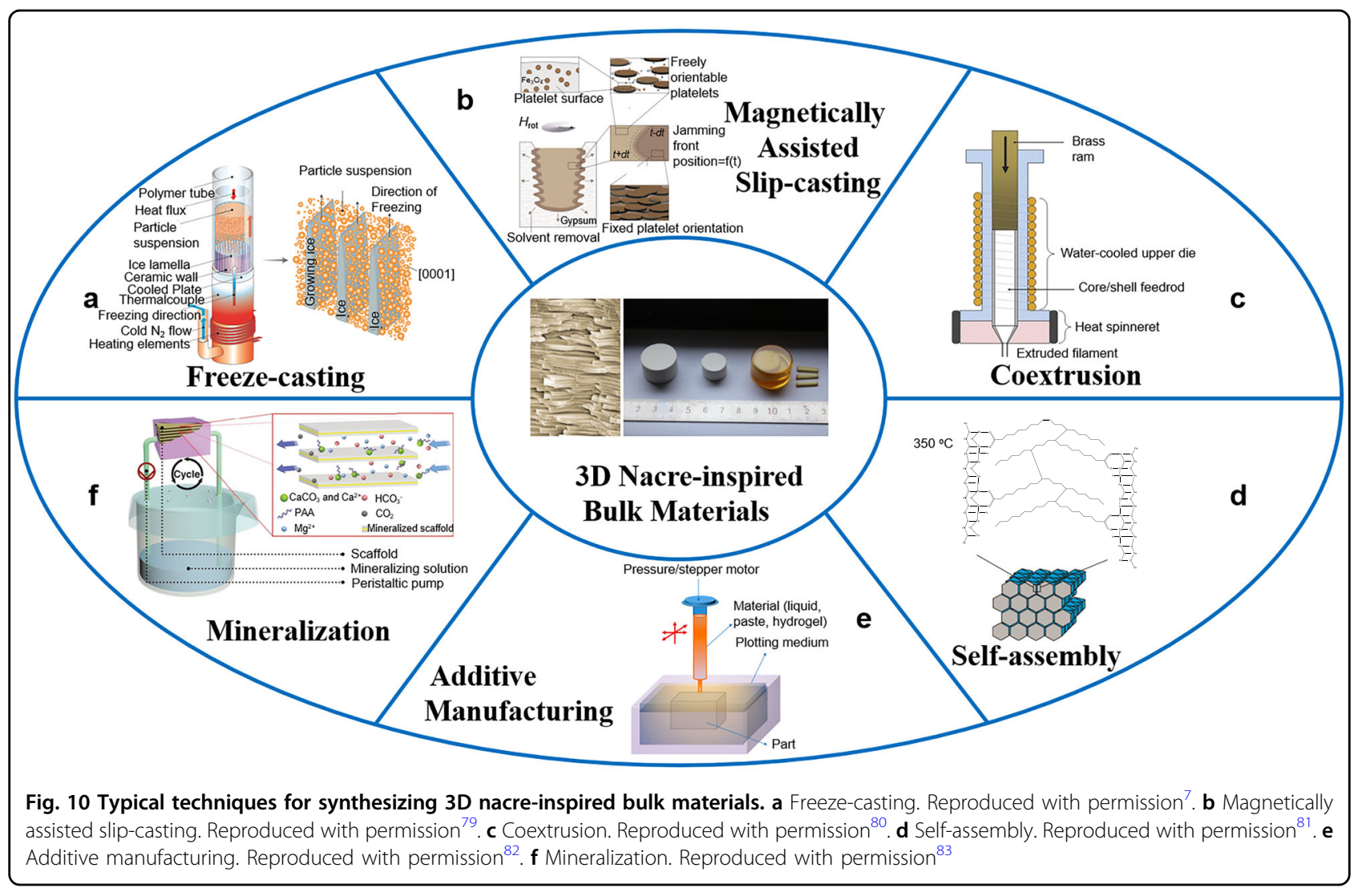

that time ${ }^{60}$. Recently, a nacre-like MTM-PVA film crosslinked by GA exhibited a fracture strength of up to $315 \mathrm{MPa}$ and showed good barrier and flame-retardant properties simultaneously when coated with polylactic acid (PLA) ${ }^{63}$.

As discussed in the section on 1D nacre-inspired fibers, interfacial interactions can be improved by introducing ions into the hierarchical layered systems. Similarly, incorporating ions into 2D layered films can also crosslink the interlayers by forming additional chemical bonds. Chen et al. ${ }^{64}$ fabricated a typical class of artificial nacre mimics by assembling hybrid graphene oxide/carboxymethylcellulose (GO/CMC) building blocks and then intercalating three different types of metal ions $\left(\mathrm{M}^{\mathrm{n}+}\right)$ via the vacuum-assisted filtration (VAF) method (Fig. 7f). Owing to the crosslinking between $\mathrm{M}^{\mathrm{n}+}$ and the oxygen (O)-containing groups of the two components, layers with metal ions (Fig. 7h) were compacted compared to those without ions (Fig. 7g). Different ions generated different bonding energies between $\mathrm{M}^{\mathrm{n}+}$ and $\mathrm{O}$, which leaded to various enhancements in mechanical properties. The bonding energy between $\mathrm{Al}^{3+}$ and $\mathrm{O}$ was strong; thus, the film reinforced by $\mathrm{Al}^{3+}$ presented an outstanding tensile strength of $300 \mathrm{MPa}$. In contrast, the zigzag long-chain structure of, for example, $(\mathrm{TiO})_{\mathrm{n}}{ }^{2 \mathrm{n}+}$ toughened the film and generated an excellent improvement in toughness of up to $20 \mathrm{MJ} / \mathrm{m}^{3}$. The synergistic crosslinking induced by metal ions in other systems such as chitosan (CS), poly (methyl methacrylate) (PMMA), and chitosan/montmorillonite (CS/MTM) was also investigated by Chen et al., and the universality of ionic crosslinking has been demonstrated ${ }^{65}$. Recently, Wan et al. ${ }^{66}$ introduced $\mathrm{Ni}^{2+}$ chelate into graphene-based nacre-like films (rGO-PDA$\mathrm{Ni}$ ), the strength and toughness of which were up to 420 $\mathrm{MPa}$ and $19 \mathrm{MJ} / \mathrm{m}^{3}$, respectively, owing to the much stronger bonding between the $\mathrm{Ni}^{2+}$ chelate and $\mathrm{O} . \mathrm{Mu}$ et $\mathrm{al}^{67}$. recently synthesized a GO-CMC nacre-like film reinforced by borate, which then triggered a strong crosslinking reaction between $\mathrm{GO}$ and $\mathrm{CMC}$, resulting in improvements in fracture strength and toughness of up to $480.5 \mathrm{MPa}$ and $11.8 \mathrm{MJ} / \mathrm{m}^{3}$, respectively. In addition, artificial nacre with shape-memory properties can be developed by introducing $\mathrm{Ca}^{2+}$ into an MTM-alginate system $^{68}$.

\section{Nanofiber reinforcement}

Incorporating the synergistic interactions from 1D nanofibers and $B M$ structures is another approach to achieve an improvement in artificial layered films, which is based on the existence of 1D nanofibrillar chitin in nacre ${ }^{17}$. Studies on ternary artificial composites have 
Table 1 Mechanical performance and functional properties of nacre-inspired composites with different macroscopic dimensions

\begin{tabular}{|c|c|c|c|c|c|c|}
\hline Dimensions & Components & Strength $(\mathrm{MPa})$ & Toughness & Performance enhanced strategy & Functional properties & Reference \\
\hline \multirow[t]{7}{*}{ 1D Fibers } & rGO-chitosan & 442 & $4.8 \mathrm{~J} / \mathrm{g}$ & Polymer crosslinking & - & 39 \\
\hline & GO-HPG-GA & 652 & $14 \mathrm{MJ} / \mathrm{m}^{3}$ & & Electrical conductivity & 40 \\
\hline & $\mathrm{rGO}-\mathrm{Ca}^{2+}$ & 501 & $6.7 \%$ & Ions crosslinking & Electrical conductivity & 42 \\
\hline & $\mathrm{rGO}-\mathrm{Cu}^{2+}$ & 408 & $6.0 \%$ & & Electrical conductivity & 42 \\
\hline & $\mathrm{rGO}-\mathrm{Ca}^{2+}-\mathrm{PCDO}$ & 842 & $16 \mathrm{MJ} / \mathrm{m}^{3}$ & & Electrical conductivity & 44 \\
\hline & $\mathrm{GO}$ & 30 & $80 \%$ & Twisting & Electrical conductivity & 47 \\
\hline & GO-PVA & 300 & $400 \%$ & & Elasticity & 48 \\
\hline \multirow[t]{18}{*}{ 2D films } & rGO-PDA & 204 & $4 \mathrm{MJ} / \mathrm{m}^{3}$ & Interface crosslinking & Electrical conductivity & 59 \\
\hline & rGO-SL & 300 & $2.8 \mathrm{MJ} / \mathrm{m}^{3}$ & & Electrical conductivity & 60 \\
\hline & Hectorite-PVA & 40 & $31 \mathrm{MJ} / \mathrm{m}^{3}$ & & Gas barrier & 58 \\
\hline & MTM-PVA-GA & 248 & $1 \%$ & & Fire-retardant & 52 \\
\hline & MTM-PVA-GA & 315 & $0.5 \%$ & & Barrier and fire-retardant & 63 \\
\hline & rGO-AP-DSS & 538.8 & $16.1 \mathrm{MJ} / \mathrm{m}^{3}$ & & Electrical conductivity & 62 \\
\hline & GO-CMC-Al $\left.\right|^{3+}$ & 304 & $5.4 \mathrm{MJ} / \mathrm{m}^{3}$ & & - & 64 \\
\hline & GO-CMC-TiO ${ }^{2+}$ & 228 & $18.9 \mathrm{MJ} / \mathrm{m}^{3}$ & & - & 64 \\
\hline & rGO-PDA-Ni & 420 & $19 \mathrm{MJ} / \mathrm{m}^{3}$ & & Electrical conductivity & 66 \\
\hline & GO-borate-CMC & 480.5 & $11.8 \mathrm{MJ} / \mathrm{m}^{3}$ & & Fire-retardant & 67 \\
\hline & MTM-ALG-Ca ${ }^{2+}$ & 280 & $7.2 \mathrm{MJ} / \mathrm{m}^{3}$ & & Fire-retardant & 68 \\
\hline & MTM-NFC-PVA & 302 & $3.72 \mathrm{MJ} / \mathrm{m}^{3}$ & Nanofiber reinforcement & Fatigue-resistant & 53 \\
\hline & SPN-NFC-TEMPO & 420 & $10 \%$ & & - & 69 \\
\hline & rGO-DWNT-PCDO & 374 & $9.2 \mathrm{MJ} / \mathrm{m}^{3}$ & & Fatigue-resistant & 70 \\
\hline & rGO-MoS 2 -TPU & 235 & $6.9 \mathrm{MJ} / \mathrm{m}^{3}$ & Nanosheets reinforcement & Electrical conductivity & 73 \\
\hline & rGO-MMT-PVA & 356 & $7.5 \mathrm{MJ} / \mathrm{m}^{3}$ & & Fire-retardant & 74 \\
\hline & GO-AA-SCMC & 305 & $8.2 \mathrm{MJ} / \mathrm{m}^{3}$ & & - & 76 \\
\hline & $\mathrm{rGO}-\mathrm{WS}_{2}-\mathrm{PCDO}$ & 413.6 & $17.7 \mathrm{MJ} / \mathrm{m}^{3}$ & & Electrical conductivity & 75 \\
\hline \multirow[t]{8}{*}{ 3D bulk materials } & $\mathrm{Al}_{2} \mathrm{O}_{3}-\mathrm{CE}$ & 300 & $5 \%$ & Bridges reinforcement & Shock-resistant & 89 \\
\hline & $\mathrm{Al}_{2} \mathrm{O}_{3}-\mathrm{SiO}_{2}$ & 650 & $14 \mathrm{MPa}^{*} \mathrm{~m}^{0.5}$ & & - & 79 \\
\hline & $\mathrm{Al}_{2} \mathrm{O}_{3}-\mathrm{PMMA}$ & 320 & $30 \mathrm{MPa}^{*} \mathrm{~m}^{0.5}$ & High content of hard phase & - & 93 \\
\hline & $\mathrm{Al}_{2} \mathrm{O}_{3}-\mathrm{PVA}$ & 320 & $40 \mathrm{MPa}^{*} \mathrm{~m}^{0.5}$ & & - & 93 \\
\hline & $\mathrm{Al}_{2} \mathrm{O}_{3}$-glasses & 470 & $22 \mathrm{MPa}^{*} \mathrm{~m}^{0.5}$ & & - & 95 \\
\hline & $\mathrm{CaCO}_{3}-\mathrm{SL}$ & 64 & $0.38 \%$ & & Bio-mineralization & 83 \\
\hline & Iron oxide-oleic acid & 620 & $0.7 \%$ & Interface modifying & - & 81 \\
\hline & Borosilicate-PU & 11.5 & $20 \%$ & & - & 105 \\
\hline
\end{tabular}

demonstrated the synergistic effects between two building blocks, such as 1D nanotubes and 2D GO nanosheets.

Wang et al. $^{53}$ adopted nanofibrillar cellulose as the third phase to synthesize a MTM-NFC-PVA ternary nacre-like artificial film via an evaporation method (Fig. 8a). The film was self-supporting, transparent and bendable (Fig. 8b).
The morphology of the ternary artificial nacre is shown in Fig. 8c, d. NFCs could be easily distinguished in the crosssection and were evenly distributed in interfaces between the MTM and PVA layers (Fig. 8d). The NFC-reinforced clay-based nacre-like film exhibited a combination of high strength and high toughness, i.e., up to $302 \mathrm{MPa}$ and 3.72 

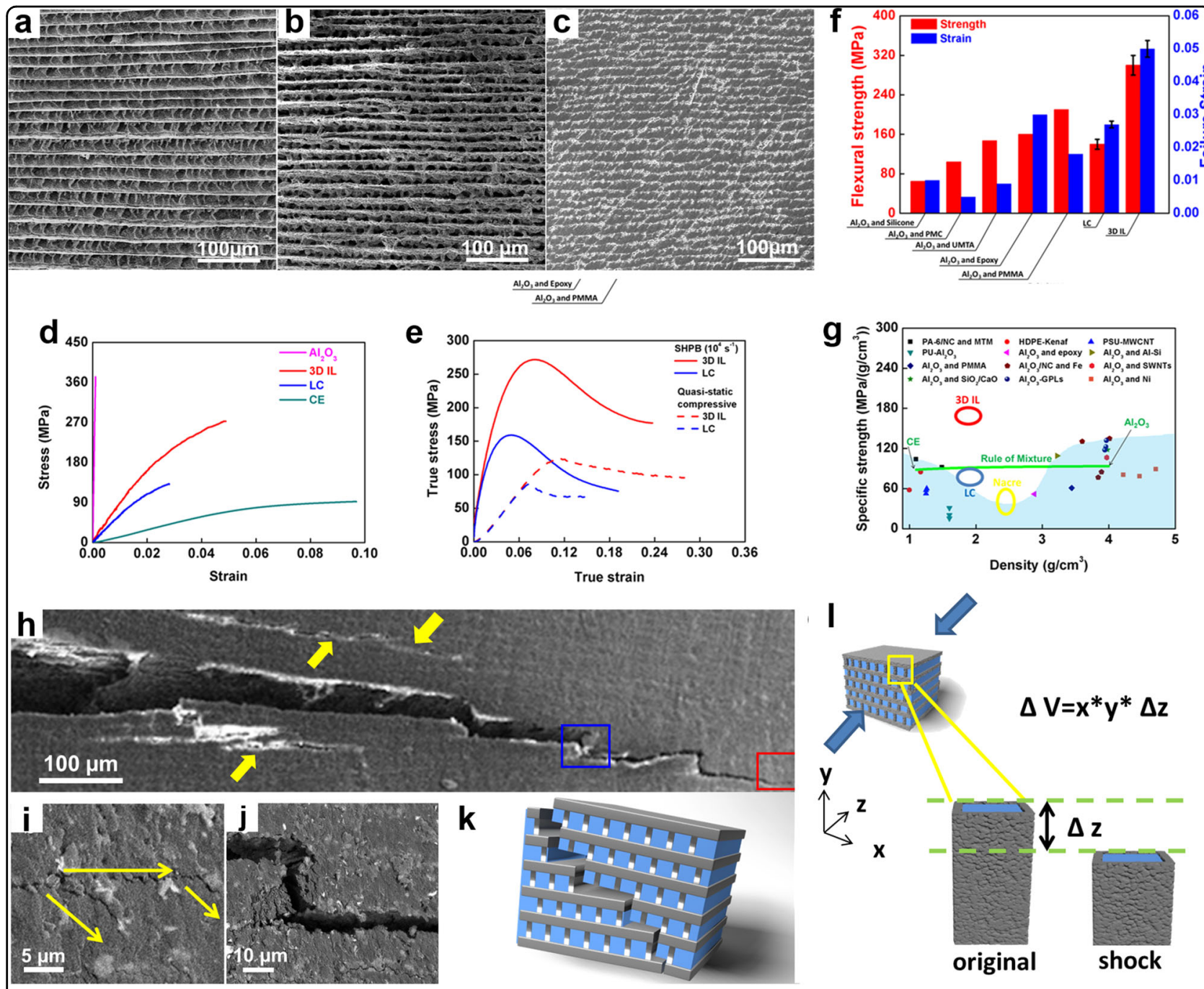

Fig. 11 3D nacre-inspired bulk materials reinforced by ordered bridges. Morphology of $\mathbf{a}$ freeze-dried samples, $\mathbf{b}$ sintered samples, and $\mathbf{c} C E$ interfiled composites, all of which show a bridge-reinforced lamellar structure (also called a 3D interlocking structure). $\mathbf{d}$ Typical bending stress-strain curves. e Typical compressive stress-strain curves from both quasi-static and dynamic tests. $\mathbf{f}$ A summary of the flexural strength and strain of 3D IL, LC, and reference bulk ceramic/polymer composites. $\mathbf{g}$ A summary of the specific strength and density of alumina-based composites. $\mathbf{h}-\mathbf{j}$ Crack propagation process observed in situ by SEM. $\mathbf{k}$ Sketch of the toughening mechanism under quasi-static conditions. I Sketch of the strengthening mechanism under dynamic conditions. Reproduced with permission ${ }^{89}$

$\mathrm{MJ} / \mathrm{m}^{3}$, respectively (Fig. 8e), which are two times higher than those of nacre. Compared to that of binary films such as MTM-PVA or NFC-PVA, the mechanical performance of ternary artificial films was higher, further demonstrating the advantages of synergistic reinforcement effect of 1D nanofibers and 2D nanosheets. In addition, the ternary film showed a fatigue-resistant property, with its strength decreasing only approximately $19 \%$ after over 10,000 cycles (Fig. 8f). A crack extension model including crack bridging, crack deflection and platelet pull-out was proposed to explain the strengthening and toughening mechanisms. In addition, Isogai et al. modified nanofibrillar cellulose with 2,2,6,6-tetramethylpiperidinyl-1-oxyl
(TEMPO) and combined it with saponite (SPN) platelets to form ternary artificial nacre exhibiting a tensile strength of $420 \mathrm{MPa}$ and strain-to-failure of $10 \%{ }^{69}$.

$1 \mathrm{D}$ carbon nanotubes (CNs) with a modulus of $1 \mathrm{TPa}$ are another building block commonly used to reinforce nacre-like films. Gong et al $^{70}$ reported an integrated strong and tough graphene-based nacre-like film reinforced by double-walled carbon nanotubes (DWNTs). The synthetic process is illustrated in Fig. 8g. GO and DWNTs were first assembled into a layered structure via an evaporation method. Then, PCDO was grafted to the $\mathrm{GO}$ and crosslinked by the polymerization of their diacetylenic units under UV irradiation. After reduction of 


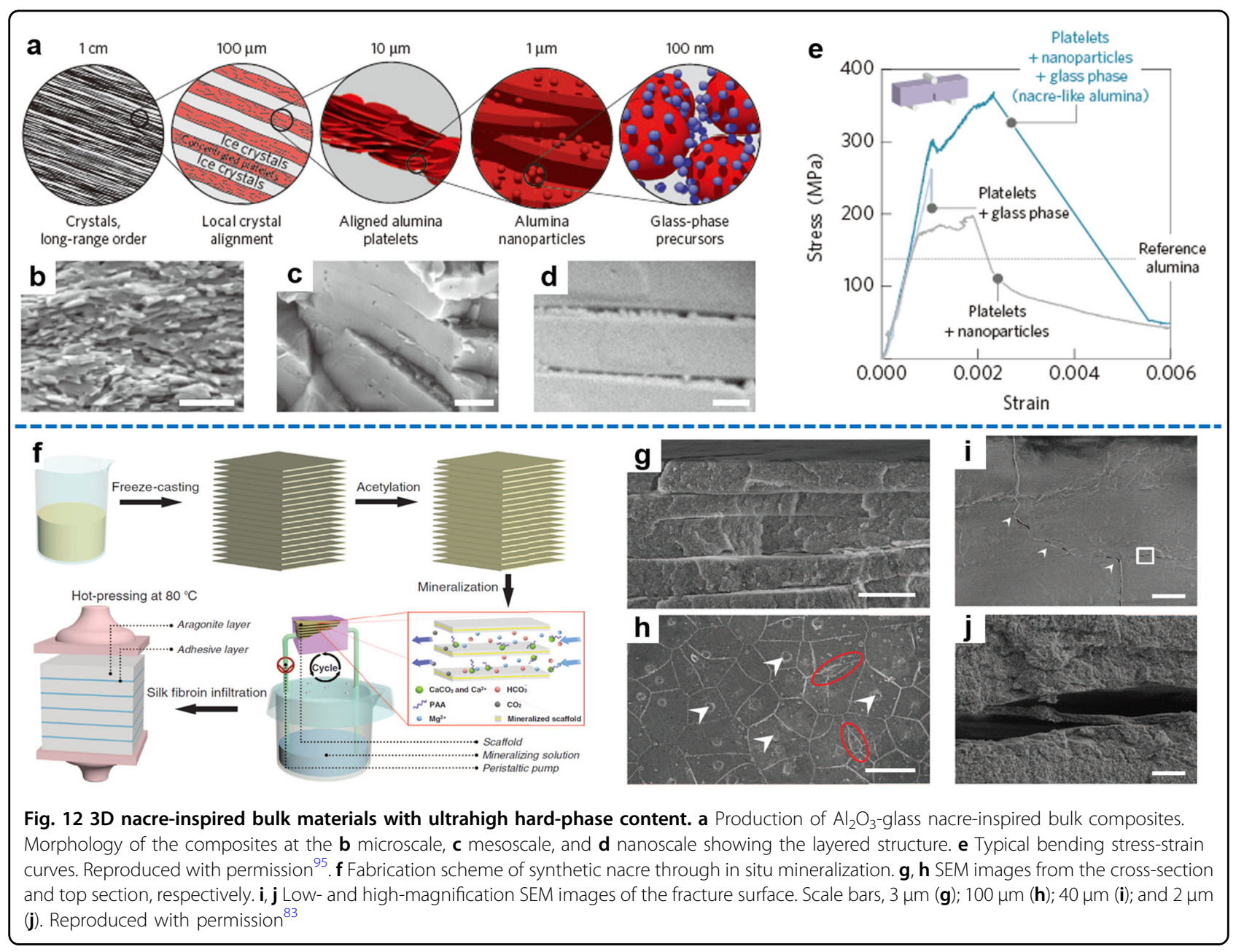

GO by HI, the final rGO-DWNT-PCDO ternary nacrelike film was formed, the morphology of which is shown in Fig. 8h, i. DWNTs were uniformly distributed in the film and were bridging the rGO and PCDO layers. The ternary graphene-based films reinforced by DWNTs showed high mechanical properties with a tensile strength of $374.1 \mathrm{MPa}$ and a toughness of $9.2 \mathrm{MJ} / \mathrm{m}^{3}$, superior to those of similar binary nacre-inspired films (Fig. 8j) owing to the strong reinforcement effect of the DWNTs. Other $1 \mathrm{D}$ nanofibers, such as vanadium pentoxide $\left(\mathrm{V}_{2} \mathrm{O}_{5}\right)^{71}$ and zirconium diboride ${ }^{72}$ nanofibers, were also used to achieve enhanced mechanical properties. Specifically, ternary artificial nacre reinforced by $\mathrm{V}_{2} \mathrm{O}_{5}$ nanofibers showed good shape-memory performance as well as excellent strength and toughness owing to the novel synergistic effects of the $1 \mathrm{D}$ and $2 \mathrm{D}$ blocks.

\section{Nanosheet reinforcement}

The addition of 2D nanosheet building blocks can also be used to enhance the mechanical performance of nacrelike films. Clay nanosheets such as MTM and disulfides such as $\mathrm{MoS}_{2}$ and $\mathrm{WS}_{2}$ have been demonstrated to effectively strengthen and toughen nacre-inspired films ${ }^{73-}$ ${ }^{75}$. For instance, Wan et al. ${ }^{73}$ introduced $\mathrm{MoS}_{2}$ nanosheets to synthesize an rGO-based ternary artificial nacre film $\left(\mathrm{rGO}-\mathrm{MoS}_{2}\right.$-thermoplastic polyurethane (TPU)) via the VAF method. The $\mathrm{MoS}_{2}$ nanosheets were lubricated and strong, making them easy to slide but hard to break and generating crack deflection during the crack propagation. As expected, the mechanical properties of the ternary nacre-inspired films were indeed improved compared to those of the binary films, exhibiting a strength and toughness of $235 \mathrm{MPa}$ and $6.9 \mathrm{MJ} / \mathrm{m}^{3}$, respectively.

The nanosheet-reinforced ternary artificial nacre film mentioned above was prepared according to the strategy of adding the reinforcing nanosheets randomly into a traditional binary system, which partially decreased the uniformity of the layered structure. Recently, Guo et al. ${ }^{76}$ successfully grew amorphous alumina nanosheets (AA) in situ on the surface of GO, and the hybrid nanosheets (GO@AA) were subsequently assembled into a layered film via the VAF method (Fig. 9a). The film was self- 


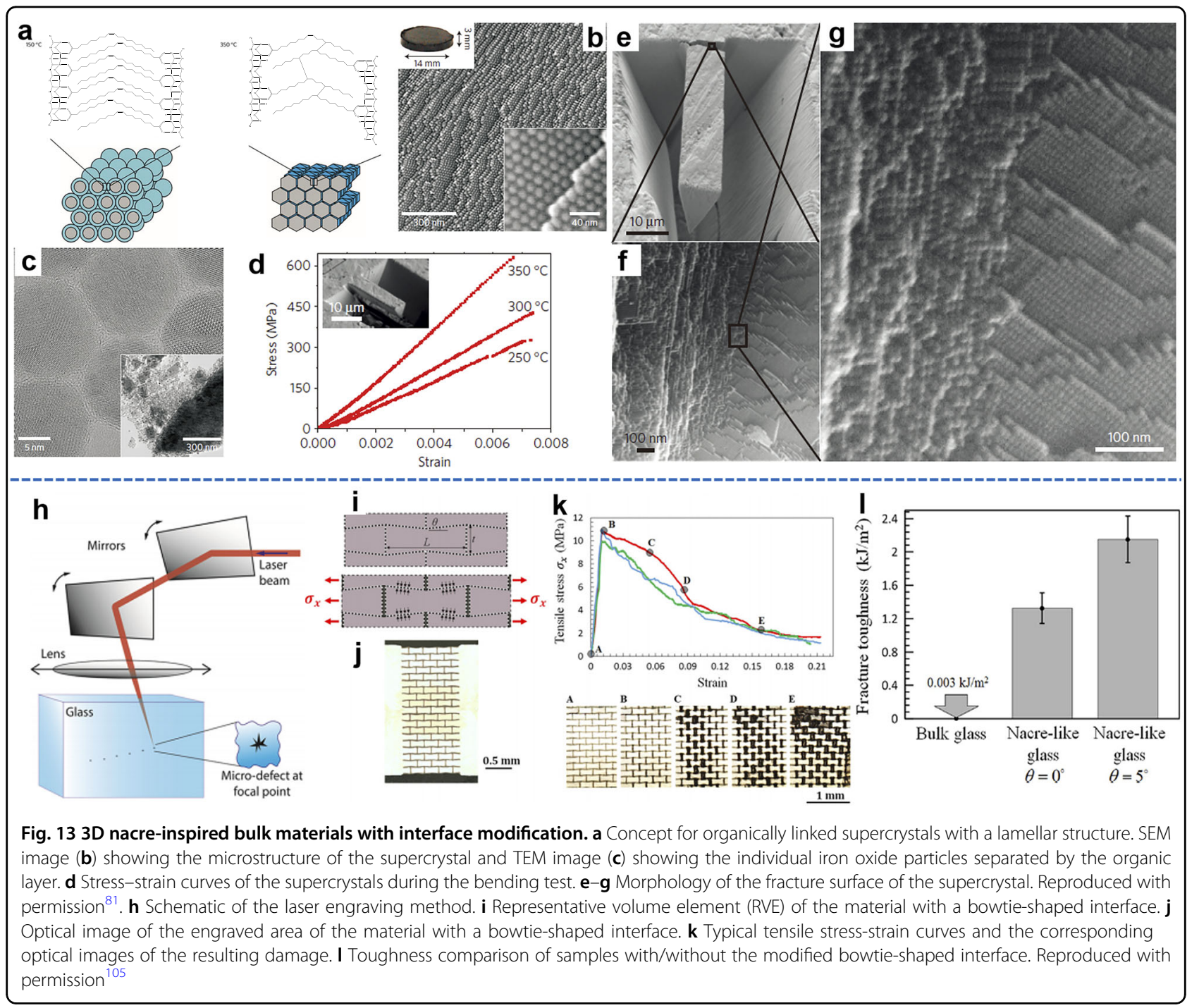

supporting and bendable (Fig. 9b). The ternary film exhibited a very ordered layered structure (Fig. 9c), and the AA nanosheets were evenly distributed in the interface between the GO and polymer layers (Fig. 9d). The uniform ternary film showed a good combination of high strength (305 MPa) and high toughness $\left(8.2 \mathrm{MJ} / \mathrm{m}^{3}\right)$, which were 2.3 and 4.3 times higher than those of nacre, respectively, representing a record strength and toughness at that time (Fig. 9f). A crack-propagation mode, including pull-out and long-range crack deflection, was proposed here to explain the outstanding mechanical properties. The surface morphology at tensile fracture was rugged (Fig. 4g, h), which confirmed the "pull-out" mode. The formation of this long-range crack deflection (Fig. 4i, j) during crack propagation can be attributed to the restrictions imposed by the AA layer on the sliding properties of the GO and the reinforcement of the stiffness of the GO@AA layer. This study provided a new path to synthesize ternary artificial nacre by the ordered assembly of three components instead of by random assembly.

Recently, Wagner et al. synthesized a Laponite-PVA lamellar film through LBL assembly and reinforced it by incorporating GO nanosheets and GA-induced chemical crosslinking simultaneously. The mechanical properties of the crosslinked ternary artificial nacre were much improved compared to those of the original binary film ${ }^{77}$.

\section{D nacre-inspired bulk materials}

3D bulk materials are used everywhere in human society owing to their tractable character, including building blocks for construction and wood for furniture. Lighter, stronger and tougher have always been the targets in the development of bulk structural materials ${ }^{29}$. Considering that nacre's exceptional mechanical properties are generated by its brick-and-mortar structure, incorporating its 


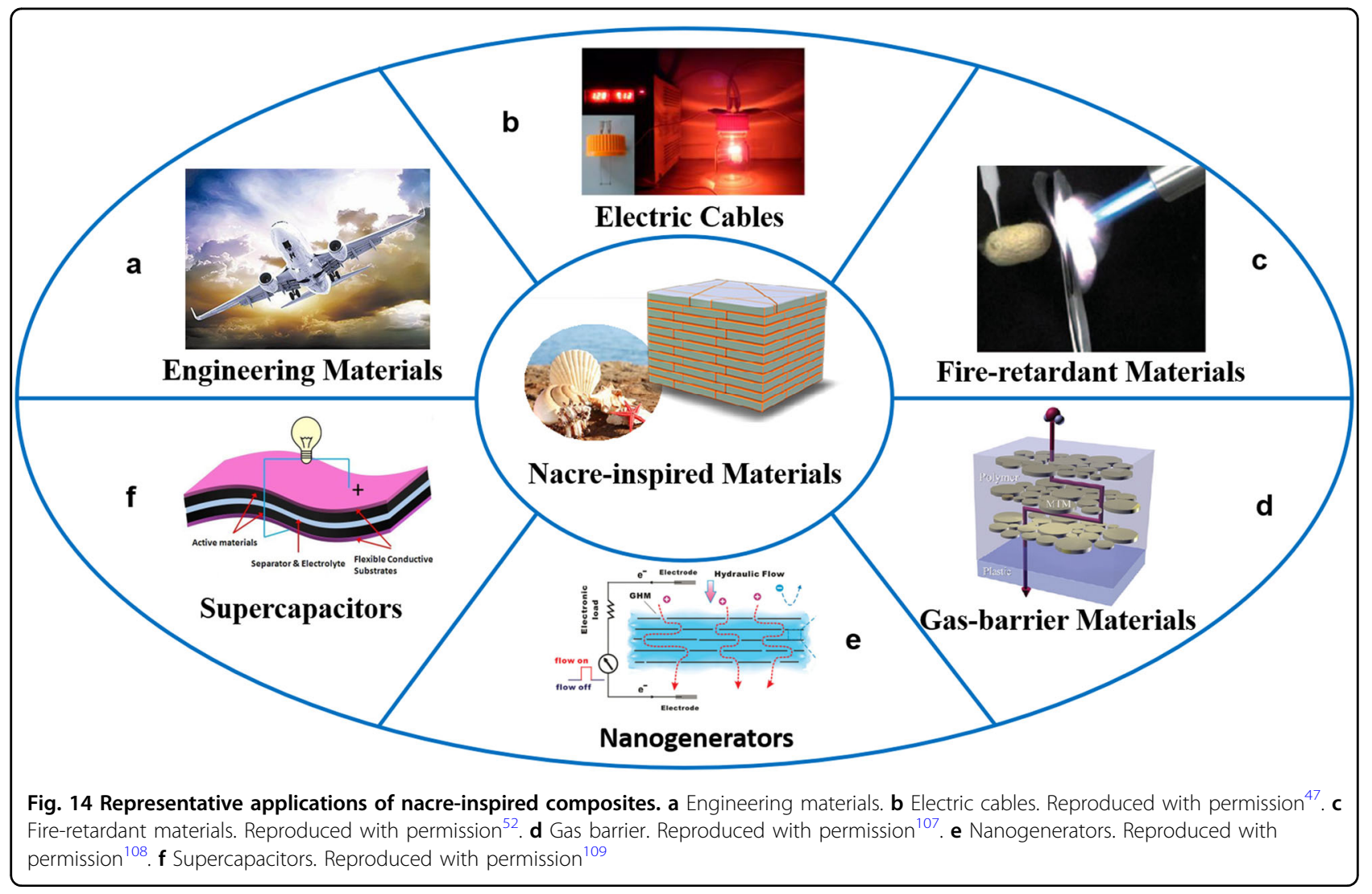

ordered architecture into bulk materials provides us with an approach for novel structural materials with better mechanical performance.

Constructing a layered structure in bulk materials was first achieved by assembling some macroscopic tablets such as $\mathrm{SiC}$ and $\mathrm{Si}_{3} \mathrm{~N}_{4}$ into layered composites through pressing, tape casting or slip-casting methods ${ }^{8,78}$. However, the layer thickness of traditional layered materials was at the macroscale (dozens of millimeters), 3-5 orders of magnitudes thicker than that of nacre (hundreds of nanometers), which extremely limited improvements in their mechanical properties. Recently, some new assembly methods, such as freeze-casting, magnetically assisted slip-casting (MASC), coextrusion, self-assembly, additive manufacturing and mineralization, have been developed (Fig. 10) to synthesize nacre-inspired bulk materials with more compact microstructures,79-83. Inspired by the freezing process of sea water, Tomsia et al. invented a directional freezing process (freeze-casting) to assemble nanoparticles into a target lamellar architecture (Fig. 10a) ${ }^{84}$. Introducing a magnetic field during the traditional slip-casting method (MASC) allowed control of the orientation of superparamagnetic nanoparticle-coated nanoplatelets to form heterogeneous lamellar microstructures (Fig. 10b), which has been demonstrated by Studart et al. $^{79}$. Coextrusion is another alternative approach to synthesize bulk lamellar composites efficiently ${ }^{80}$. Specifically, a feed rod of material was produced with specific core and shell diameters; the aspect ratio was preserved during extrusion as the rod cross-section is reduced down to a filament. The filament was then sectioned into individual pieces, representing individual "bricks" in a brick-and-mortar structure (Fig. 10c) ${ }^{80}$. Recently, iron oxide nanoparticles were self-assembled into organic molecule-oriented supercrystals with a lamellar structure (Fig. 10d), which show exceptional mechanical properties ${ }^{81}$. However, the concern with those methods is that the fabricated microstructure is not as uniform as that of nacre. Additive manufacturing methods (Fig. 10e) such as 3D printing and laser engraving were employed to synthesize nacre-like bulk materials with a more regular lamellar architecture, as they were pre-programmed ${ }^{82}$. Recently, mineralization (Fig. 10f) mimicking nacre's biomineralization process was investigated to fabricate nacre-inspired bulk composites, which could generate highly controllable structures throughout the whole bulk materials. The most obvious advantage of this method is that the prepared brick-and-mortar structure is the most similar to that of nacre from the nano- to macroscale ${ }^{83}$.

Inspired by the mineral bridges, $95 \%$ fraction of $\mathrm{CaCO}_{3}$ platelets and hierarchical interfacial interaction, 


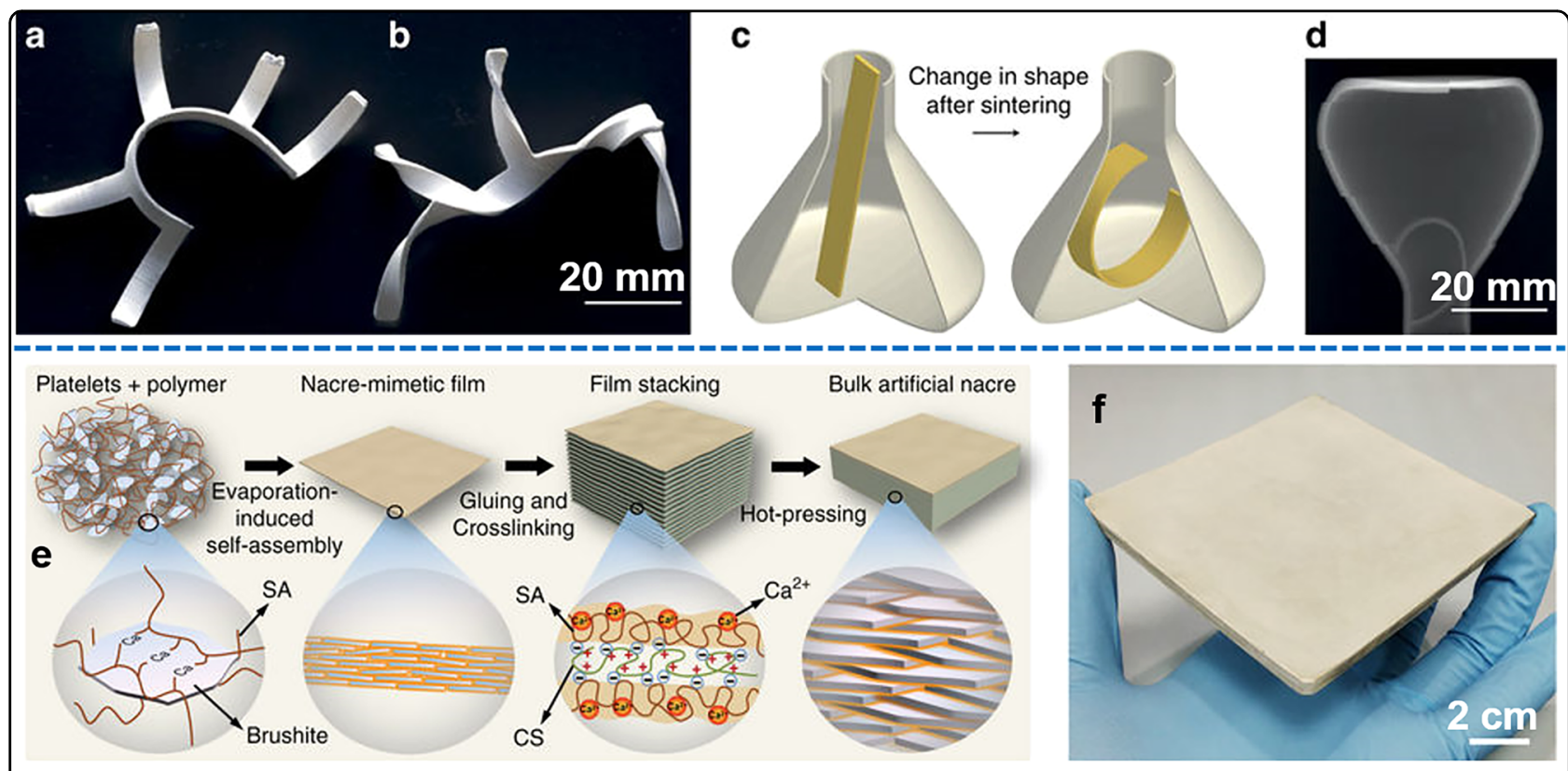

Fig. 15 Development goals of nacre-inspired materials in macroscopic size. a-d Bio-inspired ceramics with complex shapes. Reproduced with permission ${ }^{113}$. e, f Mass production of nacre-inspired bulk materials. Reproduced with permission ${ }^{114}$

introducing ordered bridges, increasing the content of the hard phase and modifying the interface in nacre-inspired bulk materials have been demonstrated to effectively promote their mechanical performance, which are reviewed in detail in the following section.

\section{Bridges reinforcement}

Mineral bridges, which play a critical role in developing nacre's impressive mechanical attributes, were first investigated in 2001 by Song et al. ${ }^{19}$. Freeze-casting is a feasible method to fabricate inorganic bridges between lamellar scaffolds by trapping inorganic particles with growing ice ${ }^{85,86}$. The introduction of inorganic bridges in nacre-inspired bulk materials could indeed promote their mechanical properties, which has also been demonstrated by Ritchie and Tomsia's studies ${ }^{87,88}$. However, due to the intermittence of the trapping of the inorganic particles by the growing ice through the traditional freeze-casting method, the bridges fabricated above were infrequent and random, which limited the reinforcement of the bridges. Recently, Guo et al. ${ }^{89}$ developed a modified ice-templating method to synthesize a lamellar alumina skeleton with ordered alumina bridges (also called a 3D interlocking skeleton). Introducing SCMC to control the movement speed of alumina nanoparticles as well as the ice-freezing process was the key to fabricating ordered bridges connected to the lamellae, called a 3D interlocking structure (3D IL). The morphology of the skeleton before (Fig. 11a) and after (Fig. 11b) sintering clearly showed a uniform 3D IL structure. After combination with a cyanate ester (CE), a bridge-reinforced $\mathrm{Al}_{2} \mathrm{O}_{3}$ - $\mathrm{CE}$ bulk composite was formed
(Fig. 11c). The composite showed a high flexural strength of $\sim 300 \mathrm{MPa}$ along with a remarkable fracture strain of $\sim 5 \%$ (Fig. 11d). The strength of the composite was approximatively equal to that of alumina tablets (360 $\mathrm{MPa}$ ), while the fracture strain was 50 times higher than that of alumina tablets $(0.1 \%)$, as shown in Fig. 11d. In addition, a standard split Hopkinson pressure bar (SHPB) system was employed to test the shock resistance of the composites, as shown in Fig. 11e. Clearly, the 3D IL sample is notable in both the quasi-static and dynamic compressive tests, and the dynamic loading fracture strength $(280 \mathrm{MPa})$ of the 3D IL sample is much higher than that under quasi-static loading (125 MPa), indicating that the 3D IL sample was indeed shock resistant. Furthermore, the failure strength of the 3D IL sample at high shock speed $\left(10^{4} / \mathrm{s}\right)$ is approximately two times greater that of the LC composite (150 MPa). Owing to the strong support of the bridge-reinforced lamellar structure, the 3D IL composite was the best combination of strength and toughness compared to similar inorganic/organic composites and exhibited the highest specific strength among alumina-based bulk composites. Long-range crack deflection and breaking of alumina bridges (Fig. 11h-j) along with multiple cracks, crack bridging and layer sliding (Fig. 11h) contributed to the high quasi-static mechanical performance. The ultra-strong support reinforcement (Fig. 11l) provided by the 3D IL structure was the reason for the enhancement in dynamic properties.

The MASC method is another efficient way to introduce inorganic bridges into nacre-inspired bulk materials owing to the sintering of precoated ceramic 
nanoparticles ${ }^{79,90,91}$. For instance, Studart et al. ${ }^{79}$ coated alumina platelets with silica or alumina particles associated with superparamagnetic iron oxide nanoparticles. After the particle-coated alumina platelets were assembled to a lamellar structure through the MASC method, cold or hot pressing was conducted on the MASC product to sinter the silica or alumina particles on the surface of opposing adjacent platelets to form bridges. As a result, the bridge-reinforced alumina-based lamellar composites show a marvelous combination of flexural strength and toughness, i.e., $650 \mathrm{MPa}$ and $14 \mathrm{MPa}^{*} \mathrm{~m}^{0.5}$, respectively. Moreover, the influence of different fractions of the mineral bridges in the nacre-inspired composites was also investigated by Studart et al. ${ }^{92}$. They successfully synthesized alumina-epoxy lamellar composites with different contents of titania bridges and demonstrated that a greater fraction of mineral bridges would result in stronger, stiffer, and tougher composites.

\section{High hard-phase content}

The hard phase $\left(\mathrm{CaCO}_{3}\right.$ platelets) in nacre mainly contributes to nacre's strength improvement, and the content of the hard phase in nacre is up to $95 \%$. Thus, increasing the hard phase content in 3D nacre-inspired composites could promote their mechanical performance, especially their strength. The lamellar composites with high hard-phase content were first obtained through layer-by-layer hand-lay-up macroscopic slabs ${ }^{93,94}$. For instance, Schneider and Wagner et al. ${ }^{93}$ increased the content of alumina in nacre-inspired composites to $90 \%$ by coating a $1-2-\mu \mathrm{m}$-thick layer of PMMA or PVA on the surface of alumina slabs with a thickness of $0.25-1.25 \mathrm{~mm}$ and subsequently holding them together. The fracture strength of the composite was increased to $\sim 320 \mathrm{MPa}$, although its much thicker layer than that of natural nacre $\left(\mathrm{CaCO}_{3}, 500-600 \mathrm{~nm}\right.$ and protein, $\left.10-20 \mathrm{~nm}\right)$ greatly limited the performance improvement.

To maximize the advantages of high hard-phase content, it is necessary to decrease the size of the lamellar structure to the submicron scale. Deville et al. ${ }^{95}$ have successfully synthesized alumina-based nacre-inspired composites with an $\sim 98 \%$ content of alumina (higher than that of natural nacre) through freeze-casting and subsequent thermal-pressing (Fig. 12a), providing an alumina layer thickness of $\sim 500 \mathrm{~nm}$, similar to that of nacre. In addition, glasses (silica-calcia liquid phase) were used as the soft phase instead of polymers to further promote the mechanical properties. SEM images are shown in Fig. 12b-d. The flexural strength of the composites exhibited an ultrahigh value of $\sim 470 \mathrm{MPa}$, which was almost the same as that of pure alumina, while the toughness was up to $22 \mathrm{MPa}^{*} \mathrm{~m}^{0.5}$ (Fig. 12e), which is five times higher than that of pure alumina. The aluminabased composite mimics nacre not only with regard to its architecture but also with regard to its micro/nanosize and hard phase content.

Controllable mineralization is of high interest because it can generate highly tunable microstructures in the macrorange and with high hard phase content ${ }^{96}$. Proteins existing in nacre such as Pif97 ${ }^{97}, \mathrm{AP}^{98}, \mathrm{n} 16.3^{99}$, PFMG $^{100}$, AP24 $4^{101}$, and Pif80 ${ }^{102}$ were considered vital to control the nucleation and formation of calcium carbonate at early stages during the mineralization process in nacre. Recently, Evans and Cölfen et al. have creatively investigated the synergistic influence of various proteins including AP7 and PEMG1 under mineralization and optimized the ratio ${ }^{103}$. With a deeper understanding of the mineralization of nacre, $\mathrm{Yu}$ and Cölfen et al. ${ }^{83}$ innovatively mimicked nacre's biomineralization in synthetic materials. In particular, they synthesized a chitosan lamellar scaffold via an ice-templating method, and $\mathrm{Ca}^{2+}$ was subsequently mineralized on the surface of the scaffold to form a lamellar $\mathrm{CaCO}_{3}$ scaffold through numerous cycles (Fig. 12f). $\mathrm{CaCO}_{3}$-silk fibroin composites with a $91 \%$ content of $\mathrm{CaCO}_{3}$ were fabricated (Figs. 12g,h) after interfiling and hot pressing the silk fibroin; the structure of these composites was the closest to that of nacre to date. As a result, the mechanical behaviors of the $\mathrm{CaCO}_{3}$ based artificial nacre, such as crack deflection, crack branching (Fig. 12i) and interlamellar debonding (Fig. 12j), was almost the same as that in nacre.

\section{Interface modification}

Similar to the enhancement strategies in 1D fibers and 2D films, modifying the interfaces between different phases in nacre-inspired bulk materials could also improve their mechanical performance. Ritchie et al. grafted methacrylate onto ceramic surfaces before PMMA inter-filtration to promote stronger covalent bonding between the two phases, and the flexural strength of the grafted sample was almost two times higher than that of the non-grafted one ${ }^{87}$.

Recently, mimicking nacre's lamellar structure in supercrystals has been achieved by Schneider et al., and the mechanical properties were much improved after interface modification ${ }^{81}$. Specifically, spherical iron oxide nanoparticles were coated with oleic acid and then selfassembled into supercrystals by slow evaporation of the solvent (Fig. 13a). After pressing and thermal treatment at $350{ }^{\circ} \mathrm{C}$, the oleic acid was crosslinked, and faceting of the iron oxide nanoparticles was started (Fig. 13a). The lamellar structure can be easily distinguished (Fig. 13b), and the iron oxide nanoparticles were separated by the organic layer (Fig. 13c). The bending mechanical test showed that the flexural strength of oleic acid-crosslinked composites (annealed at $350^{\circ} \mathrm{C}$ ) reached $630 \mathrm{MPa}$, which was two times higher than that of samples without crosslinking (annealed at $250^{\circ} \mathrm{C}$ ) (Fig. 13d). The 
observation of only a few grain boundaries without pores and cracks on the fracture surface of the nacre-like composites (Fig. 13e-g) demonstrated the importance of interface modification, which led to improved mechanical properties.

In addition to modifying the chemical state of the interfaces between two phases, the geometry of the interface can also be modified through additive manufacturing methods such as laser engraving ${ }^{104,105}$. For instance, Barthelat et $\mathrm{al}^{105}$. engraved borosilicate glass using a 3D laser engraving method, in which a UV laser was directed and focused at predefined points in space using a set of mirrors and a focusing lens (Fig. 13h). After polyurethane (PU) was interfiled into an interface engraved by the UV laser, a nacre-like bulk glass were formed. By controlling the program, the interface geometry could be manipulated into a bowtie (Fig. 13i, j) to introduce additional resistance to deformation and prevent the localization of the strains. As a result, the nacrelike bulk glass showed a remarkable fracture strain, which was up to $20 \%$ (Fig. 13k). Huge amounts of crack deformation could be observed (Fig. 13k) during the loading process, which replicated the toughening mechanism of nacre. Owing to interface modification, the glasses with a bowtie-like interface showed a 1.5 times higher toughness than those with a flat interface (Fig. 13l).

\section{Applications}

Nacre-inspired composites with different macroscopic dimensions show exceptional mechanical properties as reviewed above; therefore, they represent alternatives for replacing traditional engineering materials, such as alloys, plastics, and ceramics (Fig. 14a ${ }^{106}$. In addition, by modifying high mechanical performance materials, nacre-inspired composites exhibit potential applications in fields including electric cables, fire-retardant materials, gas barriers, nanogenerators, and supercapacitors (Fig. 14b-f) ${ }^{47,52,107-109}$.

1D nacre-inspired graphene-based fibers showing a combination of high mechanical performance and electric properties are suitable for applications in electricity transformation. For instance, after thermal treatment at $2800^{\circ} \mathrm{C}$, the twisted graphene-based nacre-like fibers showed an ultrahigh conductivity of $416 \mathrm{~S} / \mathrm{cm}^{47}$. Zhang et al. $^{44}$. reported an annealed graphene-based 1D fiber with both a high strength $(378 \mathrm{MPa})$ and a high conductivity $(285 \mathrm{~S} / \mathrm{cm})$.

Some 2D nacre-inspired clay/ceramic-based films show good physical barrier properties, such as fire-retardant ${ }^{52}$ and gas-barrier characteristics ${ }^{10,111}$. For instance, Walther et al. reported an MTM-based nacre-like films with excellent fire-shielding properties. The film kept its shape after exposure to fire (Fig. 14c) ${ }^{52}$. The ordered layered structure could prevent oxygen from transferring into the composite and reduce fuel transport into a flame, thereby weakening the combustion. Ebina et $\mathrm{al}^{110}$. synthesized a flexible transparent clay-based film with high gas-barrier properties, showing an oxygen permeability as low as $0.074 \mathrm{~cm}^{3} / \mathrm{m}^{2} /$ day $/$ atm. The key factor for its gas-barrier properties was its alignment parallel to the substrate, which can extend the diffusion path of gas and decrease the gas transmission rate. In addition, sulfonated aniline trimer (SAT)-coated GO-epoxy nacre-like films showed enhanced corrosion protection owing to the longer and more circuitous diffusion pathway of the corrosive medium generated by the lamellar structure and the excellent solvent solubility induced by the $\mathrm{SAT}^{112}$.

Some 2D nacre-inspired graphene-based films show potential applications in nanodevices and flexible supercapacitors. For instance, Guo et al. ${ }^{108}$ fabricated a 2D nanofluidic nanogenerator based on a bio-inspired graphene-based hydrogel film, which was an efficient device for converting mechanical/thermal energy into electricity. Yang et al. ${ }^{109}$ synthesized a chemically converted graphene (CCG)-based hydrogel film with high supercapacitor performance. Water was demonstrated here to serve as a spacer, preventing the restacking of CCG, which led to the high supercapacitor performance.

3D nacre-inspired bulk materials show excellent performance in terms of their light weight, strength and toughness, making them suitable for use as engineering materials. For instance, the bridge-reinforced alumina-CE composite showed a flexural strength comparable to that of steel but a density that is $\sim 1.8 \mathrm{~g} / \mathrm{cm}^{3}$, a quarter of that of steel ${ }^{89,106}$. Replacing steel with these novel composites in industry is a potential approach to achieve industrial weight loss.

\section{CONCLUSION AND OUTLOOK}

Mimicking nacre's hierarchical brick-and-mortar structure in artificial materials is an efficient approach to achieve high mechanical performance structural materials. Since 2003, when the first nacre-inspired 2D film was synthesized via the LBL method, numerous nacre-inspired composites, including 1D fibers, 2D films, and 3D bulk materials, have been fabricated. In this review, we summarize the recent achievements in nacreinspired composites classified by different dimensions and focus on novel strategies for further improving their mechanical properties. Although distinctions can be obviously seen between different cases of preparing nacreinspired composites, it is abundantly clear that the enhancement strategies can be classified according to the following two aspects: promoting interfacial interactions and tuning the nano/microstructure. Specifically, for 1D fibers, polymer crosslinking and ionic crosslinking are considered the primary strategies for enhancing performance, as they are efficient in enhancing interfacial 
interactions; other strategies include twisting, making fibers more ordered and decreasing their defects. For 2D films, according to the concept of interface design, synergetic effects generated by interfacial crosslinking, nanofiber reinforcement and nanosheet reinforcement are often adopted to improve mechanical performance. For 3D bulk materials, reinforcing the structure by introducing bridges, increasing the hard phase content and improving interfacial interactions by modifying the interface are alternative approaches for optimizing the mechanical properties. The mechanical performance and functional properties of the recently reported macroscopic nacre-inspired composites are summarized in Table 1. The mechanical properties of almost all of them are superior to those of nacre.

Although the mechanical properties of nacre-inspired artificial materials can be improved through the strategies mentioned above, replicating all the structural features of nacre simultaneously at multiple scales, including polymer behavior and interfacial interactions at the molecular level, bridges at the nanoscale, lamellar scaffolds at the microscale and high hard-phase content at the macroscopic scale, remains challenge. To combat this, novel strategies that combine increasing interfacial interactions and tuning the micro/nanostructure are necessary to synthesize macroscopic materials with a much closer nacre-mimetic architecture.

While we prepared our review of the development of nacre-inspired composites, we noticed that more literature reports on $2 \mathrm{D}$ films have been published than those on $1 \mathrm{D}$ and $3 \mathrm{D}$ materials. A possible reason is that $1 \mathrm{D}$ macroscopic structures would partially damage the lamellar structure, while for 3D materials, it is difficult to guarantee the regularity of the whole lamellar structure. When considering the incorporation of a uniform brickand-mortar structure into a target material, this challenge cannot be ignored, especially for those materials with a complicated macroscopic shape, such as the turbine blade of an aeroengine. Recently, complex-shape ceramics (Fig. 15a-d) were obtained by self-shaping via magnetic alignment and programmed heat treatment ${ }^{113}$, although the relatively irregular microstructure of complex-shape ceramics compared to that of nacre limits their mechanical performance. Thus, the synthesis of uniform nacreinspired materials with a target shape, especially those with a large and complex shape, is urgently need for further progress.

Mass production is a prerequisite of applications of nacre-inspired composites, but to date, it has been still hard to achieve until now. Recently, Yu and Cölfen et al. have successfully developed a method to synthesize largesize 3D nacre-inspired bulk composites by laminating prefabricated two-dimensional nacre-mimetic films (Fig. 15e, f) ${ }^{114}$. Although this strategy has no size restriction or fundamental barrier for further scale-up, the size of the synthesized materials was several centimeters in the lab, which is far from meeting the demands of industrial application. As a result, simplifying and modifying the synthesis strategy to make them much more suitable for scaling up the production of nacre-inspired composites with a uniform microstructure will continue to attract the interests of scientists and engineers.

In summary, nacre-inspired composites with different macroscopic dimensions provide a new approach for high mechanical performance structural materials as well as some unique functional materials. By increasing our understanding of the relationship between structure and properties, materials with better performance will be achieved, and their applications will be broadened in the near future.

\section{Acknowledgements}

This work was supported by the National Natural Science Foundation of China (51532001and 51772011), the National Basic Research Program of China (2014CB931802), the 111 Project (B14009), the National Postdoctoral Program for Innovative Talents (BX201700017), and the China Postdoctoral Science Foundation Funded Project (2017M620573).

\section{Conflict of interest}

The authors declare that they have no conflict of interest.

\section{Publisher's note}

Springer Nature remains neutral with regard to jurisdictional claims in published maps and institutional affiliations.

Received: 7 July 2017 Revised: 30 October 2017 Accepted: 3 December 2017.

Published online: 2 April 2018

\section{References}

1. Mayer, G. Rigid biological systems as models for synthetic composites. Science 310, 1144-1147 (2005).

2. $\mathrm{Yu}, \mathrm{M}$.F. et al. Strength and breaking mechanism of multiwalled carbon nanotubes under tensile load. Science 287, 637-640 (2000).

3. Lee, C., Wei, X., Kysar, J. W. \& Hone, J. Measurement of the elastic properties and intrinsic strength of monolayer graphene. Science 321, 385-388 (2008).

4. Liu, Y. H. et al. Super plastic bulk metallic glasses at room temperature. Science 315, 1385-1388 (2007).

5. Bauer, J., Hengsbach, S., Tesari, I., Schwaiger, R. \& Kraft, O. High-strength cellular ceramic composites with 3D microarchitecture. Proc. Natl Acad. Sci. USA 111, 2453-2458 (2014).

6. Zhang, C., McAdams, D. A. II \& Grunlan, J. C. Nano/micro-manufacturing of bioinspired materials: a review of methods to mimic natural structures. Adv. Mater. 28, 6292-6321 (2016).

7. Wegst, U. G. K., Bai, H., Saiz, E., Tomsia, A. P. \& Ritchie, R. O. Bioinspired structural materials. Nat. Mater. 14, 23-36 (2015).

8. Wang, J., Cheng, Q. \& Tang, Z. Layered nanocomposites inspired by the structure and mechanical properties of nacre. Chem. Soc. Rev. 41, 1111-1129 (2012).

9. Yao, H.-B., Ge, J., Mao, L.-B., Yan, Y.-X. \& Yu, S.-H. 25th anniversary article: artificial carbonate nanocrystals and layered structural nanocomposites inspired by nacre: synthesis, fabrication and applications. Adv. Mater. 26 163-188 (2014).

10. Luz, G. M. \& Mano, J. F. Biomimetic design of materials and biomaterials inspired by the structure of nacre. Philos. T. Roy. Soc. A 367, 1587-1605 (2009). 
11. Rodrigues, J. R., Alves, N. M. \& Mano, J. F. Nacre-inspired nanocomposites produced using layer-by-layer assembly: design strategies and biomedical applications. Mat. Sci. Eng. C 76, 1263-1273 (2017).

12. Mayer, G. New toughening concepts for ceramic composites from rigid natural materials. J. Mech. Behav. Biomed. 4, 670-681 (2011).

13. Meyers, M. A., McKittrick, J. \& Chen, P.-Y. Structural biological materials: critical mechanics-materials connections. Science 339, 773-779 (2013).

14. Zhang, Y. et al. Graphene-based artificial nacre nanocomposites. Chem. Soc. Rev. 45, 2378-2395 (2016).

15. Barthelat, F., Yin, Z. \& Buehler, M. J. Structure and mechanics of interfaces in biological materials. Nat. Rev. Mater. 1, 16007 (2016).

16. Studart, A. R. Additive manufacturing of biologically-inspired materials. Chem Soc. Rev. 45, 359-376 (2016).

17. Wang, R. Z., Suo, Z., Evans, A. G., Yao, N. \& Aksay, I. A. Deformation mechanisms in nacre. J. Mater. Res. 16, 2485-2493 (2001).

18. Li, X., Xu, Z.H. \& Wang, R. In situ observation of nanograin rotation and deformation in nacre. Nano Lett. 6, 2301-2304 (2006).

19. Song, F., Soh, A. K. \& Bai, Y. L. Structural and mechanical properties of the organic matrix layers of nacre. Biomaterials 24, 3623-3631 (2003).

20. Barthelat, F. \& Espinosa, H. D. An experimental investigation of deformation and fracture of nacre-mother of pearl. Exp. Mech. 47, 311-324 (2007).

21. Menig, R., Meyers, M. H., Meyers, M. A. \& Vecchio, K. S. Quasi-static and dynamic mechanical response of Haliotis rufescens (abalone) shells. Acta Mater. 48, 2383-2398 (2000).

22. Li, X., Chang, W.-C., Chao, Y. J., Wang, R. \& Chang, M. Nanoscale structural and mechanical characterization of a natural nanocomposite Material: the shell of red abalone. Nano Lett. 4, 613-617 (2004).

23. Jackson, A. P., Vincent, J. F. V. \& Turner, R. M. The mechanical design of nacre P. Roy. Soc. B Biol. Sci. 234, 415-440 (1988).

24. Nukala, P. K. V. V. \& Simunovic, S. A continuous damage random thresholds model for simulating the fracture behavior of nacre. Biomaterials $\mathbf{2 6}$ 6087-6098 (2005).

25. Meyers, M. A., Chen, P. Y., Lin, A. Y. M. \& Seki, Y. Biological materials: structure and mechanical properties. Prog. Mater. Sci. 53, 1-206 (2008).

26. Rabiei, R., Bekah, S. \& Barthelat, F. Failure mode transition in nacre and bonelike materials. Acta Biomater. 6, 4081-4089 (2010).

27. Wagner, H. D. \& Weiner, S. On the relationship between the microstructure of bone and its mechanical stiffness. J. Biomech. 25, 1311-1320 (1992).

28. Espinosa, H. D. et al. Tablet-level origin of toughening in abalone shells and translation to synthetic composite materials. Nat. Commun. 2, 173 (2011).

29. Cruz-Silva, R., Endo, M. \& Terrones, M. Graphene oxide films, fibers, and membranes. Nanotechnol. Rev. 5, 377-391 (2016).

30. Cheng, H., Hu, C., Zhao, Y. \& Qu, L. Graphene fiber: a new material platform for unique applications. NPG Asia Mater. 6, e113 (2014).

31. Xu, Z. \& Gao, C. Graphene chiral liquid crystals and macroscopic assembled fibres. Nat. Commun. 2, 571 (2011).

32. Zou, J. \& Kim, F. Self-assembly of two-dimensional nanosheets induced by interfacial polyionic complexation. ACS Nano 6, 10606-10613 (2012).

33. Dong, Z. et al. Facile fabrication of light, flexible and multifunctional graphene fibers. Adv. Mater. 24, 1856-1861 (2012).

34. Li, X. et al. Multifunctional graphene woven fabrics. Sci. Rep. 2, 395 (2012).

35. Jalili, R. et al. Organic solvent-based graphene oxide liquid crystals: a facile route toward the next generation of self-assembled layer-by-layer multifunctional 3D architectures. ACS Nano 7, 3981-3990 (2013).

36. Liu, Z., Xu, Z., Hu, X. \& Gao, C. Lyotropic liquid crystal of polyacrylonitrilegrafted graphene oxide and its assembled continuous strong nacre-mimetic fibers. Macromolecules 46, 6931-6941 (2013).

37. Kou, L. \& Gao, C. Bioinspired design and macroscopic assembly of poly(vinyl alcohol)-coated graphene into kilometers-long fibers. Nanoscale $\mathbf{5}$ 4370-4378 (2013).

38. Zhao, X., Xu, Z., Zheng, B. \& Gao, C. Macroscopic assembled, ultrastrong and $\mathrm{H}_{2} \mathrm{SO}_{4}$-resistant fibres of polymer-grafted graphene oxide. Sci. Rep. 3, 3164 (2013).

39. Jalili, R. et al. Scalable one-step wet-spinning of graphene fibers and yarns from liquid crystalline dispersions of graphene oxide: towards multifunctional textiles. Adv. Funct. Mater. 23, 5345-5354 (2013).

40. Hu, X., Xu, Z., Liu, Z. \& Gao, C. Liquid crystal self-templating approach to ultrastrong and tough biomimic composites. Sci. Rep. 3, 2374 (2013).

41. Weiner, S. \& Dove, P. M. An overview of biomineralization processes and the problem of the vital effect. Rev. Mineral. geochem. 54, 1-29 (2003).
42. Xu, Z., Sun, H., Zhao, X. \& Gao, C. Ultrastrong fibers assembled from giant graphene oxide sheets. Adv. Mater. 25, 188-193 (2013).

43. Hu, X., Xu, Z. \& Gao, C. Multifunctional, supramolecular, continuous artificial nacre fibres. Sci. Rep. 2, 767 (2012).

44. Zhang, Y. et al. Ultrastrong bioinspired graphene-based fibers via synergistic toughening. Adv. Mater. 28, 2834-2839 (2016).

45. Xu, Z. \& Gao, C. Graphene in macroscopic order: liquid crystals and wet-spun fibers. Acc. Chem. Res. 47, 1267-1276 (2014).

46. Zhang, M., Atkinson, K. R. \& Baughman, R. H. Multifunctional carbon nanotube yarns by downsizing an ancient technology. Science 306, 1358-1361 (2004).

47. Cruz-Silva, R. et al. Super-stretchable graphene oxide macroscopic fibers with outstanding knotability fabricated by dry film scrolling. ACS Nano $\mathbf{8}$, 5959-5967 (2014).

48. Zhang, J. et al. Multiscale deformations lead to high toughness and circularly polarized emission in helical nacre-like fibres. Nat. Commun. 7, 10701 (2016).

49. Richardson, J. J., Bjoernmalm, M. \& Caruso, F. Technology-driven layer-bylayer assembly of nanofilms. Science 348, aaa2491 (2015).

50. Bonderer, L. J., Studart, A. R. \& Gauckler, L. J. Bioinspired design and assembly of platelet reinforced polymer films. Science 319, 1069-1073 (2008).

51. Chavez-Valdez, A., Shaffer, M. S. P. \& Boccaccini, A. R. Applications of graphene electrophoretic deposition. A review. J. Phy. Chem. B 117, 1502-1515 (2013).

52. Walther, A. et al. Large-area, lightweight and thick biomimetic composites with superior material properties via fast, economic, and green pathways. Nano Lett. 10, 2742-2748 (2010).

53. Wang, J., Cheng, Q., Lin, L. \& Jiang, L. Synergistic toughening of bioinspired poly(vinyl alcohol)-clay-nanofibrillar cellulose artificial nacre. ACS Nano 8 , 2739-2745 (2014).

54. Tang, Z. Y., Kotov, N. A., Magonov, S. \& Ozturk, B. Nanostructured artificial nacre. Nat. Mater. 2, 413-418 (2003).

55. Podsiadlo, P. et al. Layer-by-layer assembly of nacre-like nanostructured composites with antimicrobial properties. Langmuir 21, 11915-11921 (2005).

56. Podsiadlo, P., Liu, Z., Paterson, D., Messersmith, P. B. \& Kotov, N. A. Fusion of seashell nacre and marine bioadhesive analogs: high-strength nanocompoisite by layer-by-layer assembly of clay and L-3,4-dihydroxyphenylaianine polymer. Adv. Mater. 19, 949-955 (2007).

57. Mirkhalaf, M. \& Barthelat, F. Nacre-like materials using a simple doctor blading technique: Fabrication, testing and modeling. J. Mech. Behav. Biomed. 56, 23-33 (2016).

58. Das, P. et al. Nacre-mimetics with synthetic nanoclays up to ultrahigh aspect ratios. Nat. Commun. 6, 5967 (2015).

59. Cui, W. et al. A strong integrated strength and toughness artificial nacre based on dopamine cross-linked graphene oxide. ACS Nano 8, 9511-9517 (2014).

60. $\mathrm{Hu}, \mathrm{K}$. et al. Written-in conductive patterns on robust graphene oxide biopaper by electrochemical microstamping. Angew. Chem. Int. Ed. 52, 13784-13788 (2013)

61. Wu, Q. et al. Facile and universal superhydrophobic modification to fabricate waterborne, multifunctional nacre-mimetic films with excellent stability. ACS Appl. Mater. Inter. 6, 20597-20602 (2014).

62. $\mathrm{Ni}, \mathrm{H}$. et al. Robust bioinspired graphene film via $\pi-\pi$ cross-linking. ACS Appl. Mater. Inter. 9, 24987-24992 (2017).

63. Ding, F. C. et al. Biomimetic nanocoatings with exceptional mechanical, barrier, and flame-retardant properties from large-scale one-step coassembly. Sci. Adv. 3, e1701212 (2017).

64. Chen, K., Tang, X., Yue, Y., Zhao, H. \& Guo, L. Strong and tough layered nanocomposites with buried interfaces. ACS Nano 10, 4816-4827 (2016).

65. Chen, $\mathrm{K}$. et al. A general bioinspired, metals-based synergic cross-linking strategy toward mechanically enhanced materials. ACS Nano 11, 2835-2845 (2017).

66. Wan, S., Xu, F., Jiang, L. \& Cheng, Q. Superior fatigue resistant bioinspired graphene-based nanocomposite via synergistic interfacial interactions. Adv Funct. Mater. 27, 1605636 (2017).

67. Shahzadi, K. et al. Bio-based artificial nacre with excellent mechanical and barrier properties realized by a facile in situ reduction and cross-linking reaction. ACS Nano 11, 325-334 (2017).

68. Liang, B. et al. Ca2+ enhanced nacre-inspired montmorillonite-alginate film with superior mechanical, transparent, fire retardancy, and shape memory properties. ACS Appl. Mater. Inter. 8, 28816-28823 (2016). 
69. Wu, C. N., Yang, Q. L., Takeuchi, M., Saito, T. \& Isogai, A. Highly tough and transparent layered composites of nanocellulose and synthetic silicate. Nanoscale 6, 392-399 (2014).

70. Gong, S. et al. Integrated ternary bioinspired nanocomposites via synergistic toughening of reduced graphene oxide and double-walled carbon nanotubes. ACS Nano 9, 11568-11573 (2015).

71. Knoeller, A. et al. Strengthening of ceramic-based artificial nacre via synergistic interactions of 1D vanadium pentoxide and 2D graphene oxide building blocks. Sci. Rep. 7, 40999 (2017).

72. An, Y. et al. Synergistic effect on the mechanical behaviors of ternary graphene oxide-zirconium diboride-poly(vinyl alcohol) papers. Mater. Des. 112 275-281 (2016).

73. Wan, S. et al. Synergistic toughening of graphene oxide-molybdenum disulfide-thermoplastic polyurethane ternary artificial nacre. ACS Nano 9 708-714 (2015).

74. Ming, P. et al. Nacre-inspired integrated nanocomposites with fire retardant properties by graphene oxide and montmorillonite. J. Mater. Chem. A $\mathbf{3}$, 21194-21200 (2015).

75. Wan, S. et al. Fatigue resistant bioinspired composite from synergistic twodimensional nanocomponents. ACS Nano 11, 7074-7083 (2017).

76. Zhao, H., Yue, Y., Zhang, Y., Li, L. \& Guo, L. Ternary artificial nacre reinforced by ultrathin amorphous alumina with exceptional mechanical properties. Adv. Mater. 28, 2037-2042 (2016).

77. Patro, T. U. \& Wagner, H. D. Influence of graphene oxide incorporation and chemical cross-linking on structure and mechanical properties of layer-bylayer assembled poly(vinylalcohol)-laponite free-standing films. J. Polym. Sci. Pol. Phys. 54, 2377-2387 (2016).

78. Padture, N. P., Pender, D. C., Wuttiphan, S. \& Lawn, B. R. In situ processing of silicon carbide layer structures. J. Am. Ceram. Soc. 78, 3160-3162 (1995).

79. Le Ferrand, H., Bouville, F., Niebel, T. P. \& Studart, A. R. Magnetically assisted slip casting of bioinspired heterogeneous composites. Nat. Mater. 14 1172-1179 (2015).

80. Wilkerson, R. P. et al. A novel approach to developing biomimetic ("nacrelike") metal-compliant-phase (nickel-alumina) ceramics through coextrusion. Adv. Mater. 28, 10061-10067 (2016).

81. Dreyer, A. et al. Organically linked iron oxide nanoparticle supercrystals with exceptional isotropic mechanical properties. Nat. Mater. 15, 522-528 (2016).

82. Yadav, R., Naebe, M., Wang, X. \& Kandasubramanian, B. Review on 3D prototyping of damage tolerant interdigitating brick arrays of nacre. Ind. Eng. Chem. Res. 56, 10516-10525 (2017).

83. Mao, L.-B. et al. Synthetic nacre by predesigned matrix-directed mineralization. Science 354, 107-110 (2016).

84. Deville, S., Saiz, E., Nalla, R. K. \& Tomsia, A. P. Freezing as a path to build complex composites. Science 311, 515-518 (2006).

85. Launey, M. E. et al. Designing highly toughened hybrid composites through nature-inspired hierarchical complexity. Acta Mater. 57, 2919-2932 (2009).

86. Zhao, N. et al. Superstretchable nacre-mimetic graphene/poly(vinyl alcohol) composite film based on interfacial architectural engineering. ACS Nano 11, 4777-4784 (2017).

87. Munch, E. et al. Tough, bio-inspired hybrid materials. Science 322, 1516-1520 (2008).

88. Launey, M. E. et al. A novel biomimetic approach to the design of high-performance ceramic-metal composites. J. R. Soc. Interface 7, 741-753 (2010)

89. Zhao, $\mathrm{H}$. et al. Cloning nacre's $3 \mathrm{D}$ interlocking skeleton in engineering composites to achieve exceptional mechanical properties. Adv. Mater. $\mathbf{2 8}$ 5099-5105 (2016).

90. Libanori, R. et al. Composites reinforced via mechanical interlocking of surface-roughened microplatelets within ductile and brittle matrices. Bioinspir. Biomim. 11, 036004 (2016).
91. Niebel, T. P., Carnelli, D., Binelli, M. R., Libanori, R. \& Studart, A. R. Hierarchically roughened microplatelets enhance the strength and ductility of nacreinspired composites. J. Mech. Behav. Biomed. 60, 367-377 (2016).

92. Grossman, M. et al. Mineral nano-interconnectivity stiffens and toughens nacre-like composite materials. Adv. Mater. 29, 1605039 (2017).

93. Livanov, K. et al. Tough alumina/polymer layered composites with high ceramic content. J. Am. Ceram. Soc. 98, 1285-1291 (2015).

94. Behr, S., Kollner, A. \& Schneider, G. A. Tailoring toughness and mechanical reliability by controlled defects: nature-inspired composite laminates of laserperforated yttria-stabilized zirconia. Adv. Eng. Mater. 18, 1877-1883 (2016).

95. Bouville, F. et al. Strong, tough and stiff bioinspired ceramics from brittle constituents. Nat. Mater. 13, 508-514 (2014).

96. Barthelat, F. Growing a synthetic mollusk shell. Science 354, 32-33 (2016).

97. Chang, E. P. \& Evans, J. S. Pif97, a von Willebrand and Peritrophin biomineralization protein, organizes mineral nanoparticles and creates intracrystalline nanochambers. Biochemistry 54, 5348-5355 (2015).

98. Perovic, I. et al. An oligomeric C-RING nacre protein influences prenucleation events and organizes mineral nanoparticles. Biochemistry 53, 7259-7268 (2014).

99. Perovic, I. et al. A nacre protein, n16.3, self-assembles to form protein oligomers that dimensionally limit and organize mineral deposits. Biochemistry 53, 2739-2748 (2014).

100. Perovic, I., Mandal, T. \& Evans, J. S. A pearl protein self-assembles to form protein complexes that amplify mineralization. Biochemistry 52, 5696-5703 (2013).

101. Chang, E. P., Perovic, I., Rao, A., Colfen, H. \& Evans, J. S. Insect cell glycosylation and its impact on the functionality of a recombinant intracrystalline nacre protein, AP24. Biochemistry 55, 1024-1035 (2016).

102. Bahn, S. Y., Jo, B. H., Choi, Y. S. \& Cha, H. J. Control of nacre biomineralization by Pif80 in pearl oyster. Sci. Adv. 3, e1700765 (2017).

103. Chang, E. P. et al. Synergistic biomineralization phenomena created by a combinatorial nacre protein model system. Biochemistry 55, 2401-2410 (2016).

104. Mirkhalaf, M., Dastjerdi, A. K. \& Barthelat, F. Overcoming the brittleness of glass through bio-inspiration and micro-architecture. Nat. Commun. 5, 3166 (2014).

105. Valashani, S. M. M. \& Barthelat, F. A laser-engraved glass duplicating the structure, mechanics and performance of natural nacre. Bioinspir. Biomim. 10 026005 (2015).

106. Ashby, M. F. Materials Selection in Mechanical Design 4th edn (Elsevier Ltd., Burlington, MA, USA, 2010).

107. Choi, J. H. et al. Fuzzy nanoassembly of polyelectrolyte and layered clay multicomposite toward a reliable gas barrier. Langmuir 28, 6826-6831 (2012).

108. Guo, W. et al. Bio-inspired two-dimensional nanofluidic generators based on a layered graphene hydrogel membrane. Adv. Mater. 25, 6064-6068 (2013).

109. Yang, X., Zhu, J., Qiu, L. \& Li, D. Bioinspired effective prevention of restacking in multilayered graphene films: towards the next generation of highperformance supercapacitors. Adv. Mater. 23, 2833-2838 (2011).

110. Ebina, T. \& Mizukami, F. Flexible transparent clay films with heat-resistant and high gas-barrier properties. Adv. Mater. 19, 2450-2453 (2007).

111. Tritschler, U. et al. Gas barrier properties of bio-inspired Laponite-LC polymer hybrid films. Bioinspir. Biomim. 11, 035005 (2016).

112. Lu, H. Zhang, S. Li, W. Cui, Y. \& Yang, T. Synthesis of graphene oxide-based sulfonated oligoanilines coatings for synergistically enhanced corrosion protection in 3.5\% NaCl solution. ACS Appl. Mater. Inter 9, 4034-4043 (2017).

113. Bargardi, F. L., Le Ferrand, H., Libanori, R. \& Studart, A. R. Bio-inspired selfshaping ceramics. Nat. Commun. 7, 13912 (2016).

114. Gao, H.-L. et al. Mass production of bulk artificial nacre with excellent mechanical properties. Nat. Commun. 8, 287 (2017) 